\title{
Late Quaternary insects and freshwater invertebrates of the Alaskan North Slope and paleoenvironmental reconstructions in Arctic Alaska
}

\author{
S.A. Kuzmina ${ }^{1 a}$, S.A. Elias ${ }^{2}$, A.A. Kotov ${ }^{3,4}$ \\ ${ }^{I}$ Laboratory of Arthropods, Borissiak Palaeontological Institute, Profsoyuznaya Str. 123, 117997, \\ Moscow, Russia. \\ ${ }^{2}$ Institute of Arctic and Alpine Research, University of Colorado, Boulder, CO 80309-0450, USA. \\ ${ }^{3}$ Laboratory for Ecology of Aquatic Communities and Invasions, A.N. Severtsov Institute of Ecology \\ and Evolution, Russian Academy of Sciences, Leninsky Prospect 33, Moscow 119071, Russia. \\ ${ }^{4}$ Laboratory of Paleoclimatology, Kazan Federal University, Kremlevskaya Street 18, Kazan \\ 420000, Russia. \\ E-mails:svkuz@yandex.ru,S.Elias@rhul.ac.uk,alexey-a-kotov@yandex.ru
}

ABSTRACT: A large series (26 samples) of fossil insect assemblages were excavated from riverbank exposures at two localities at Ikpikpuk and Titaluk Rivers on the North Slope of Alaska, U.S.A. Climatic conditions were reconstructed for the Late Pleistocene and early Holocene based on the fossil insect assemblage data. Insects indicate the continuous existence of a steppe-tundra community on the Alaskan North Slope during the end of the Pleistocene and the beginning of the Holocene. The invasion of poplar during the Early Holocene occurred within the context of the steppe-tundra community. The insect faunas indicate plant communities dominated by grasses and other herbs, with the local presence of tall shrubs and dwarf willows. The composition of the North Slope insect communities during the study interval was affected by the high latitude of the localities, periglacial winds coming off Brooks Range glaciers, and the close proximity of eolian sand and silt. The North Slope environment differed from those in more southerly localities in Eastern Beringia, reflecting ancient climatic and vegetational zonation.

How to cite this article: Kuzmina S.A., Elias S.A., Kotov A.A. 2019. Late Quaternary insects and freshwater invertebrates of the Alaskan North Slope and paleoenvironmental reconstructions in Arctic Alaska// Invert. Zool. Vol.16. No.2. P.89-125. doi: 10.15298/ invertzool.16.2.02

KEY WORDS: Pleistocene, Holocene, fossil insects, beetles, Cladocera, Beringia, steppetundra.

\section{Позднечетвертичные насекомые и пресноводные беспозвоночные Северного Склона Аляски и реконструкции природной обстановки для арктической Аляски}

\section{С.А. Кузьмина ${ }^{1 a}$, С.А. Элайс ${ }^{2}$, А.А. Котов ${ }^{3,4}$}

${ }^{1}$ Лаборатория артропод, Палеонтологический институт им. А.А. Борисяка, ул. Профсоюзная 123, 117997, Москва, Россия.

${ }^{2}$ Institute of Arctic and Alpine Research, University of Colorado, Boulder, CO 80309-0450, USA. 
3 Лаборатория экологии водных сообществ и инвазий, Инститкт проблем экологии и эволючии им. А.Н. Севериова РАН, Ленинский проспект 33, Москва 119071, Россия.

${ }^{4}$ Лаборатория палеоклиматологии, Казанский (Приволжский) федеральный университет, ул. Кремлевская 18, Казань 420000, Республика Татарстан, Россия.

E-mails:svkuz@yandex.ru,S.Elias@rhul.ac.uk, alexey-a-kotov@yandex.ru

РЕЗЮМЕ: Большая серия образцов (26 штук) с ископаемыми насекомыми была отобрана из двух разрезов на реках Икпикпук и Титалук на Северном Склоне Аляски. На основании изучения комплексов насекомых получены реконструкции климата для позднего плейстоцена и раннего голоцена. Насекомые позволяют утверждать, что ландшафты на Северном Склоне Аляски, и во время позднего плейстоцена, и в начале голоцена, были тундростепными. Даже появление на данной территории тополя в начале голоцена не отразилось на тундростепном сообществе насекомых. Энтомофауна существовала в растительном сообществе из злаков, разнотравья, локального развития среднего размера кустарников и карликовой ивы. На фауну насекомых Северного Склона оказывали влияние северное расположение района, стоковый ветер с хребта Брукса, где в то время было развито горное оледенение, и близость источника эоловых песков и пыли. Природная обстановка на Северном Склоне отличалась от того, что наблюдалось в более южных районах Восточной Беринги, что отражает существовавшую в прошлом природно-климатическую зональность.

Как цитировать эту статью: Kuzmina S.A., Elias S.A., Kotov A.A. 2019. Late Quaternary insects and freshwater invertebrates of the Alaskan North Slope and paleoenvironmental reconstructions in Arctic Alaska// Invert. Zool. Vol.16. No.2. P.89-125. doi: 10.15298/ invertzool.16.2.02

КЛЮЧЕВЫЕ СЛОВА: плейстоцен, голоцен, ископаемые насекомые, жуки, Cladocera, Берингия, тундростепь.

\section{Introduction}

The enormous ancient region known as Beringia included the northeastern part of Russia and the northwestern regions of North America, linked together by the Bering Land Bridge. Beringia played a critical role in the history of the Arctic flora and fauna (Hultén, 1937; Yurtsev, 1972; Hopkins, 1972; Sher, 1976; Matthews et al., 2019). Because of its aridity, Beringia remained mainly unglaciated throughout the Pleistocene. Thus Beringia was essentially the only Arctic region that remained ice-free, making it a refuge for Arctic flora and fauna during Pleistocene glaciations. Beringia was covered by different kinds of grasslands with a minor component of trees in regions of higher moisture. There are different opinions about the nature of Beringian vegetation cover, ranging from tundra-like environments similar to those of the modern Arctic (Colinvaux, 1967; Ager, 1982; Anderson et al., 1989), to meadow-like tundra (Anderson, 1985), a mosaic grassland (Schweger, 1997) to arid steppe-tundra (Sher, 1974; Matthews, 1979; Kiselev, 1981; Guthrie, 1990, 2001; Yurtsev, 2001; Zazula et al., 2003; Kienast, 2006). These different reconstructions are caused primarily by the different proxies used to make them. Thus, pollen studies may indicate tundra or meadow environments, while plant macrofossil, mammal and insect studies indicate steppe-tundra. The kind of environmental reconstruction also depends on the region studied. For instance, in Western Beringia, insect fossil assemblages from Late Pleistocene cold stages (MIS4, MIS2) indicate well developed steppe-tundra in the Kolyma and YanaIndigirka lowlands, while tundra-like communities with little contribution from steppe species have been identified from far northern 
Siberia (Medvezhji Islands, Bolshoy Lyakhovsky Island). Meanwhile, meadow-tundra vegetation has been reconstructed for the southern Chukotka region (Kuzmina, 2015).

The composition of vegetation zones in Western Beringia was affected by the distance from the Arctic Ocean, by the distance from glaciers, and by distance from high mountain ridges. Environmental conditions in the interior regions in Western Beringia, such as Kolyma Lowland, were favorable for the establishment of steppe vegetation. Even during coldest time of the Pleistocene, the Last Glacial Maximum (LGM), summer temperatures were higher here than they are today (Alfimov, Berman, 2001, Alfimov et al., 2003). In contrast, temperature reconstructions made from insect fossil assemblages in Eastern Beringia (Elias, 2001) indicated that summer temperatures during the LGM were lower than today.

A paleontological expedition to the North Slope of Alaska took place in June 2003 after the $3^{\text {rd }}$ International Mammoth Conference in Dawson City and Whitehorse, Yukon. Paul Matheus was a member of the conference host committee; Andrei Sher was one of distinguished guests and a session chair. During a pre-conference meeting, Paul and Andrei discussed possibility of joint fieldwork in Alaska. It was difficult bureaucratic task since the Russian members (A.V. Sher and S.A. Kuzmina) needed visas to enter Canada and USA; combining the conference with a field trip to Alaska provided a good opportunity to obtain these visas. A.V. Sher wanted to visit two sites to collect insect fossils: the Alaskan analogue of Siberian "Yedoma" on the North Slope of Alaska and a tephra buried soil on Seward Peninsula. Insect remains had already been observed at both sites (Nelson, Carter, 1987; Goetcheus et al., 1994, 2001; Goetcheus, 2001) but the sections were tested differently than the methods used for Siberian sites. Thus Sher's idea was to apply the "Russian" method of insect sampling and subsequent treatment, involving the screening of large volumes of the sediment in the field, and the sampling of entire sections at exposures (not just the most interesting layers); and, most im- portantly, the identification of the Alaskan insect remains by a Russian expert. Such methodological comparisons would be helpful in the understanding of the similarities and differences between Western and Eastern Beringia insect faunas.

The plan worked well. A.V. Sher, S.A. Kuzmina and P. Matheus collected rich fossil insect assemblages from two sites on the North Slope and one site on Seward Peninsula (Kuzmina, Elias, 2019). The results of the expedition to Seward Peninsula have been published (Kuzmina et al., 2008) but the results of the North Slope investigations have not been published until now. The authors have taken the opportunity to complete this important research that began with Andrei Sher's 2003 initiative.

\section{Regional setting}

\section{Modern environment and climate}

The North Slope of Alaska (Fig. 1) is situated between the Brooks Range and the Arctic Ocean. Today it is a treeless region of flat plains and gentle foothills (Fig. 2 A, C, D). While the North Slope itself has never been glaciated, the Brooks Range was covered by ice during LGM (Kaufman, Manley, 2004; Briner, Kaufman, 2008). These mountains contribute to windy weather on the plain. During the cold stages of the Late Pleistocene, dry winds coming off the Brooks Range helped shape the Ikpikpuk Sand Sea with its famous sand dunes (Carter, 1981). These winds also helped to accumulate loess deposits exposed along the Ikpikpuk and Titaluk rivers. A significant part of the Pleistocene deposits in the study area were formed by alluvium derived from numerous streams flowing out of the Brooks Range. The studied sections of these two rivers contain gravel and sand units with cross bedding (Fig. 3).

The recent climate of the interior regions of the North Slope is cold and relatively dry. The mean annual air temperature is $-10.2^{\circ} \mathrm{C}$, the warmest month is July $\left(12-13{ }^{\circ} \mathrm{C}\right)$, and mean annual precipitation is $122 \mathrm{~mm}$, mostly falling as snow (Kene et al., 2014). The active layers above permafrost are $15-40 \mathrm{~cm}$ thick in summer 


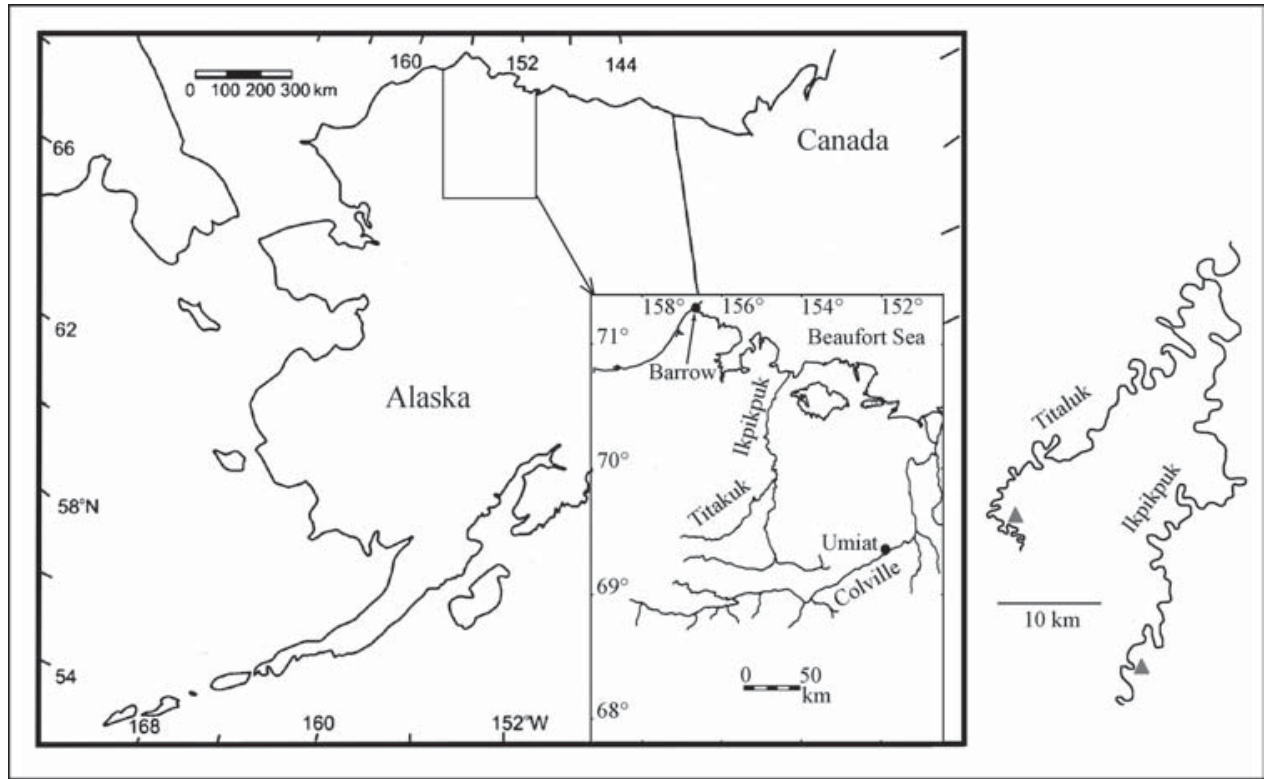

Fig. 1. Map of the study area.

Рис. 1. Карта изученного района.

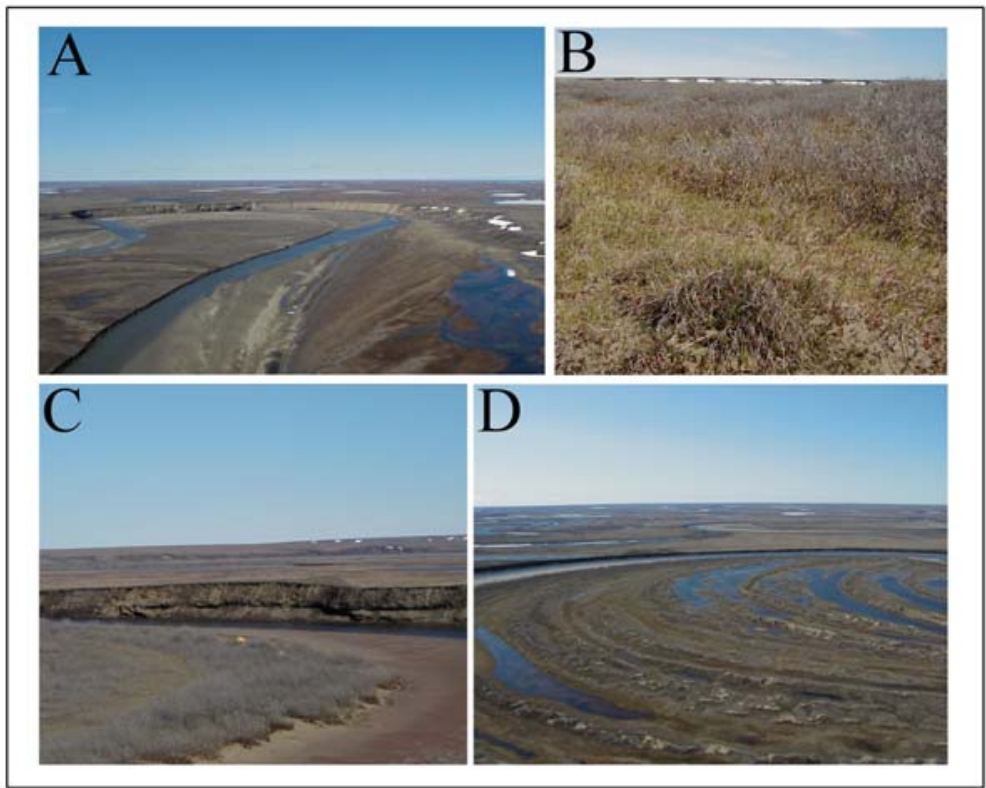

Fig. 2. Landscape and vegetation of North Slope tundra in early June 2003.

A - aerial view of the Ikpikpuk River with a river bluff, sand bars, lakes and snow; B - upland vegetation includes short willows, grasses, and sedges; the surface is covered by sand from a nearby outcrop; C - field camp on sand bar of the Titaluk River, showing belts of tall willows, boggy meadow, and shrub alder; D — spring water fills old channels in the floodplain.

Рис. 2. Ландшафт и растительность тундры Северного Склона в начале июня 2003 г.

А - вид из вертолета на реку Икпикпук, речные обрывы, песчаные отмели, озера и снег; В - растительность тундры вне долины реки с низкими ивами, травами и осоками, почва покрыта песком, надутым с ближайшего обрыва; C - полевой лагерь на песчаном пляже реки Титалук, видны полосы высокой кустарниковой растительности из ивы и ольховника, и заболоченные луга; $\mathrm{D}$ - весенняя вода заполнила ложбины в пойме реки. 


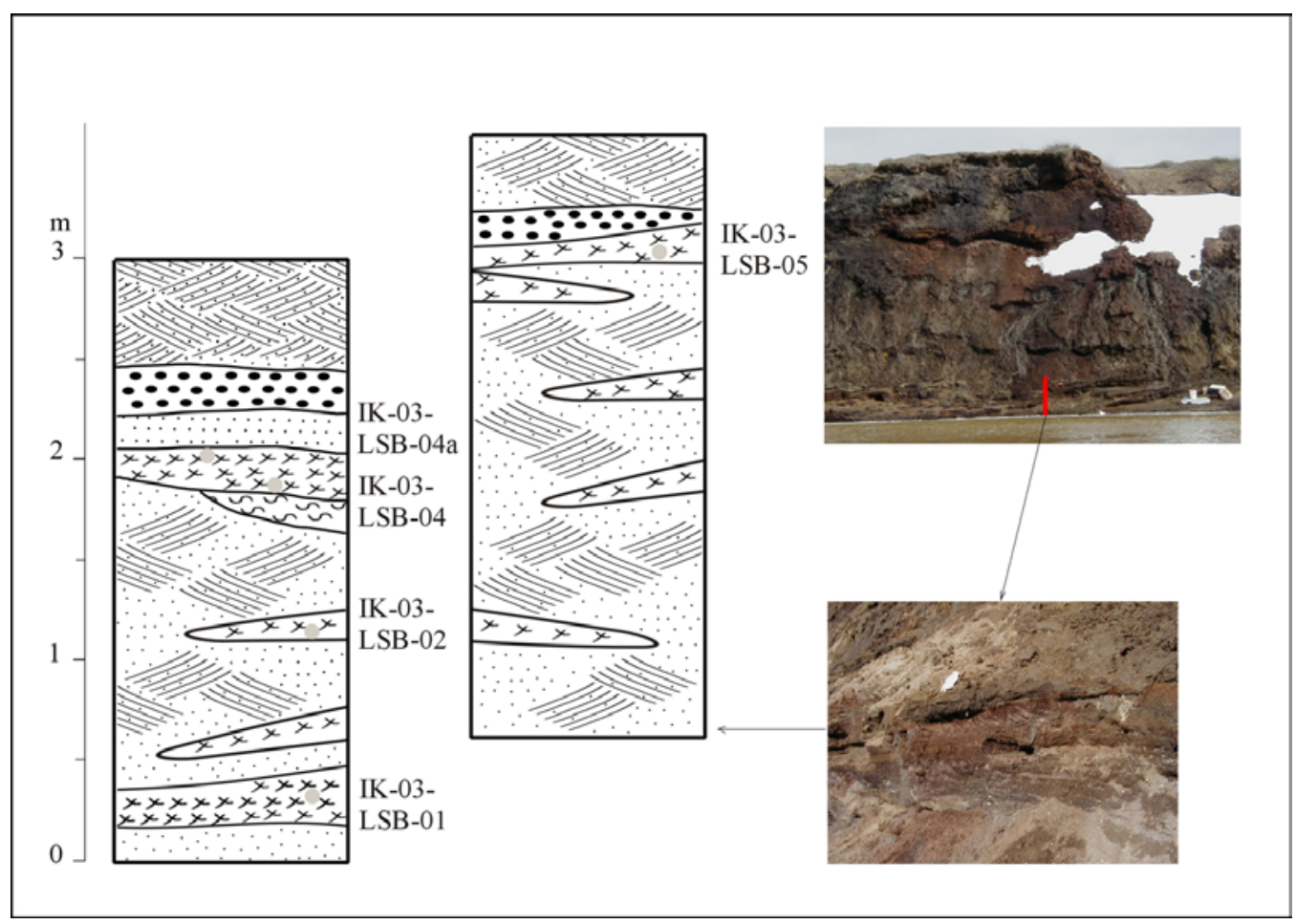

Fig. 3. Ikpikpuk Section "Little Supreme Bluff” (LSB).

Рис. 3. Разрез “маленький верховой обрыв” (ЛСБ) на реке Икпикпук.

(Bockheim, Hinkel, 2005), keeping water on the surface (Fig. 2 D) despite the low precipitation. Modern vegetation includes sedge-grass and moss tundra with dwarf shrubs. Middle sized willow and alder shrubs occur in parts of the river valleys (Fig. 2 C). Balsam poplar (Populus balsamifera L.) may grow in valleys (Bockheim et al., 2003) but these trees are absent near the studied sections.

\section{Stratigraphic framework}

The studied sections are located on the Ikpikpuk River and its tributary, the Titaluk River. The river sources lie in the foothills of the Brooks Range. Upstream channels contain Cretaceous sandstone bedrock; the eroded sandstone provides coarse and fine sand, and gravel for the river alluvium. The sands yield small pieces of amber and coal, probably of Cretaceous origin.

The Ikpikpuk sand sea (Carter, 1981), situated near confluence of the Ikpikpuk and Ti- taluk Rivers, deposited sand more-or-less continuously in and around the two rivers during the Late Pleistocene. The dune field was active between 41 and $16.7 \mathrm{cal} \mathrm{ka} \mathrm{BP}$, then between 12.9 and $8.7 \mathrm{cal} \mathrm{ka} \mathrm{BP} \mathrm{(Carter,} \mathrm{1993).} \mathrm{Wind}$ carried sand from the dune deposit to the studied sections. When the dune field was active, the local vegetation was constantly being covered by sand; mosses could not survive here, and the land surface became unusually dry (Fig. 2 B). Such xeric places increased the biotope diversity of the study area. Modern ground beetles (Carabidae) collected from interior regions of the North Slope (Nelson, 2001) include 56 species while only 9 species have been found at coastal sites. Our collection of modern beetles includes such common Pleistocene species as Lepidophorus lineaticollis Kby, 1837, Poecilus nearcticus Lindr., 1966, Stereocerus haematopus (Dej., 1831), and Amara alpina Payk., 1790. These beetles were found at the edge of the river bluff on sandy soil. 
Table 1. Radiocarbon data from the key sections of the North Slope. Таблица 1. Радиоуглеродные датировки из ключевых разрезов Северного Склона.

\begin{tabular}{|c|c|c|c|c|c|}
\hline Unit & & & ${ }^{14} \mathrm{C}$ age & & \\
\hline site & Ikpikpuk & Titaluk & Nigu & Etivluk & Itkillik \\
\hline Holo- & $2310 \pm 70 \mathrm{~b}$ & $2500 \pm 50 \mathrm{e}$ & & $4900 \pm 60 \mathrm{f}$ & $5320 \pm 35 d$ \\
\hline cene & $5430 \pm 60 \mathrm{~b}$ & & & & $8610 \pm 35 \mathrm{~d}$ \\
\hline & $6070 \pm 60 \mathrm{~b}$ & & & & \\
\hline $\mathrm{D}$ & $8710 \pm 140 \mathrm{a}$ & & $8080 \pm 50 \mathrm{~b}$ & $8360 \pm 70 \mathrm{f}$ & \\
\hline Populus & $9050 \pm 60 \mathrm{~b}$ & & & $8870 \pm 60 \mathrm{f}$ & \\
\hline Bed & $9250 \pm 70 \mathrm{~b}$ & & & $9020 \pm 50 \mathrm{f}$ & \\
\hline & $9340 \pm 60 \mathrm{~b}$ & $9450 \pm 60 \mathrm{~b}$ & & $9570 \pm 60 \mathrm{f}$ & \\
\hline & $9480 \pm 50 \mathrm{~b}$ & $9510 \pm 60 \mathrm{~b}$ & $9670 \pm 50 \mathrm{~b}$ & & \\
\hline & $9540 \pm 160 \mathrm{a}$ & & $10,920 \pm 50 \mathrm{~b}$ & & \\
\hline$C$ & & & & & $14370+100$ \\
\hline Yedoma & & & & & $16,550 \pm 75 \mathrm{~d}$ \\
\hline & & & & & $19,950 \pm 180 \mathrm{c}$ \\
\hline & & & & & $24,290 \pm 190 \mathrm{c}$ \\
\hline & & & & & $25,450 \pm 280 \mathrm{c}$ \\
\hline & & & & & $26,300 \pm 130 \mathrm{~d}$ \\
\hline & & & & & $28,330 \pm 390 \mathrm{c}$ \\
\hline & & & & & $41,700 \pm 460 \mathrm{~d}$ \\
\hline B upper & $10,230 \pm 160 \mathrm{a}$ & & & $10,470 \pm 50 \mathrm{f}$ & \\
\hline sand & $11,860 \pm 50 \mathrm{~b}$ & & $11,370 \pm 50 \mathrm{~b}$ & $12,450 \pm 60 \mathrm{f}$ & \\
\hline & $13,670 \pm 130 \mathrm{a}$ & & $12,230 \pm 50 \mathrm{~b}$ & $13,420 \pm 50 \mathrm{f}$ & \\
\hline & $17,730 \pm 110 \mathrm{a}$ & & & & \\
\hline $\mathrm{B}$ middle & $20,360 \pm 190 \mathrm{a}$ & & & & \\
\hline & $24,740 \pm 32 \mathrm{a}$ & $28,000 \pm 240 \mathrm{e}$ & & $29,570 \pm 150 \mathrm{f}$ & \\
\hline & $30,260 \pm 530 \mathrm{a}$ & & & $34,920 \pm 260 \mathrm{f}$ & \\
\hline B lower & $>39,000 \mathrm{a}$ & $43,000 \pm 1400 \mathrm{e}$ & & $47,830 \pm$ & \\
\hline sand & & & & $1300 \mathrm{f}$ & \\
\hline & $>40,000 \mathrm{a}$ & & & & \\
\hline & $>42,500 \mathrm{~b}$ & & & & \\
\hline & $>47,770 \mathrm{~b}$ & & & & \\
\hline A gravel & $>49,000 \mathrm{a}$ & & & & \\
\hline
\end{tabular}

From: Nelson, Carter, 1987 (a); Mann et al., 2010 (b); Lapointe et al., 2017 (c); Kanevskiy et al., 2011 (d); Trimble, Robinson, 1989 (e); Mann et al., 2002 (f).

Both rivers are surrounded by vast sand bars (Fig. 2 A, C) where rich Pleistocene bone assemblages have been collected (Guthrie, Stoker, 1990; Mann et al., 2010, 2013). The Pleistocene megafauna of the North Slope was dominated by mostly horses, with Pleistocene bison, caribou, woolly mammoth, and muskox, with rare specimens of moose, saiga antelope, mastodon, and predators including short-faced bear, cave lion, and wolf (Mann et al., 2010, 2013).
Most of the Pleistocene sections of the North Slope are situated along rivers. The key sections are Itkillik (Kanevskiy et al., 2011, 2016; Lapointe et al., 2017), Nigu (Mann et al., 2010), Etivluk (Mann et al, 2002; Gaglioti et al., 2017) Titaluk and Ikpikpuk (Mann et al., 2010, 2013, 2015; Gaglioti et al., 2017, 2018) and a sediment core from Burial lake (Finkenbinder et al., 2015). The river outcrops (except buried sand dunes such as Carter's section at the Ikpikpuk 
River) usually consist of fluvial deposits: gravel and sand. In some sections (Itkillik, Titaluk) the uppermost unit consists of silts with ice wedges that are similar to the Siberian "Yedoma" deposits (Kanevskiy et al., 2016). Another interesting feature is the "Populus Bed" (Nelson, Carter, 1987). There are depressions in the main Pleistocene unit filled by early Holocene sediments containing well-preserved leaves, logs and stumps of Populus balsamifera. Since the modern limit of this tree species is south of the North Slope, the presence of fossil poplar indicates warmer than present climate during the early Holocene.

\section{Radiocarbon dating}

Radiocarbon dates were obtained from several sections (Table 1). The best studied are the sections at Ikpikpuk River(Little Supreme Bluff and Cottonwood Bluff) where the main focus was dating of the Populus Bed. Lower gravel unit at Ikpikpuk River is older than $50 \mathrm{ka}$ BP (infinite radiocarbon ages). The same unit was observed at the base of the Titaluk sections. The main sand body was accumulated from ca. $40 \mathrm{ka}$ or earlier, to $10-11 \mathrm{ka} \mathrm{BP}$. The Yedoma-analogue unit was observed at the Tituluk site, and was well studied at the Itkillik site, but was absent from the Ikpikpuk site. It is likely that the sandy unit of the Ikpikpuk section yielded a nondepositional unconformity about $30 \mathrm{ka}$ BP. The age of the Titaluk Yedoma-analogue unit remains uncertain but stratigraphic correlation with the Itkillik section allows us to infer that it formed during LGM.

\section{Studied sections}

Fossil insects were collected from two sections (Fig. 1): 1 - Ikpikpuk River, Little Supreme Bluff (LSB) $\left(69^{\circ} 35^{\prime} 21^{\prime \prime} \mathrm{N}, 1^{\circ} 4^{\circ} 56^{\prime} 14^{\prime \prime}\right.$ W) and Titaluk River, Russian Section (RS) $\left(69^{\circ} 25^{\prime} 48^{\prime \prime} \mathrm{N}, 155^{\circ} 9^{\prime} 26.2^{\prime \prime} \mathrm{W}\right)$. The Carter Section at the Titaluk River (Gaglioti et al., 2018) was visited but useful layers for insect sampling were not found. Since the expedition took place in early June, the water level in the rivers was high. Only the lower part of the LSB exposure was sampled (Fig. 3); RS (Figs 4, 5) was sam- pled from all accessible units, including two Holocene buried channels with poplar leaves.

Section LSB at the Ikpikpuk River is one of the best-studied in the area (Mann et al., 2002, 2010; Gaglioti et al., 2017). Our team visited the exposure first and planned to stay there for several days but the section was poorly exposed, as the top was covered by snow (Fig. 3). We tested only lower gravel unit, the Purple Gravel of Mann et al. (2010). The unit consists of crossbeds of iron-stained pebbly gravel with plant debris and willow roots. A radiocarbon age of the unit (from willow wood) is infinite - more than $47 \mathrm{ka} \mathrm{yr}$.

The Russian Section (RS) at the Titaluk River had not been previously described. This section was better exposed (Figs 4, 5); we spent about two weeks working there. The lower part of the section is similar to LSB. There is crossbedded gravel with plant debris; the unit is traced along whole outcrop, about $1 \mathrm{~km}$ long. The deposit is exposed from the river level up to 2-2.5 m.

The gravel unit is overlaid with a visible unconformity by a sand unit. It consists of crossbeds and horizontal sand with numerous thin layers of plant debris. The plant layers were found to contain abundant fossil insect remains. The age of the unit is uncertain. Sher considered that the unit could be very old, either from the middle or early Pleistocene. Infinite radiocarbon ages from a similar sand deposit at the Cottonwood Bend Section at Ikpikpuk River (Nelson, Carter, 1987) support this assumption. However, other radiocarbon dates from 30 to 11 ka yr (Table 1) indicate that the whole unit probably belongs to the late Pleistocene. Perhaps the lower part of the sand is older.

Ice-rich silt with ice wedges are situated at the top of the section (Fig. 5). This unit is very similar to Siberian Yedoma, based on the field experience of Sher and Kuzmina; the sediment was wet because of melting, and plant inclusions were not evident. In contrast to the lower units, the silt looked fossil-free. We paid special attention to the insect sampling of the silt. Large volume screening of sediments yielded good insect assemblages from this organic-poor sed- 


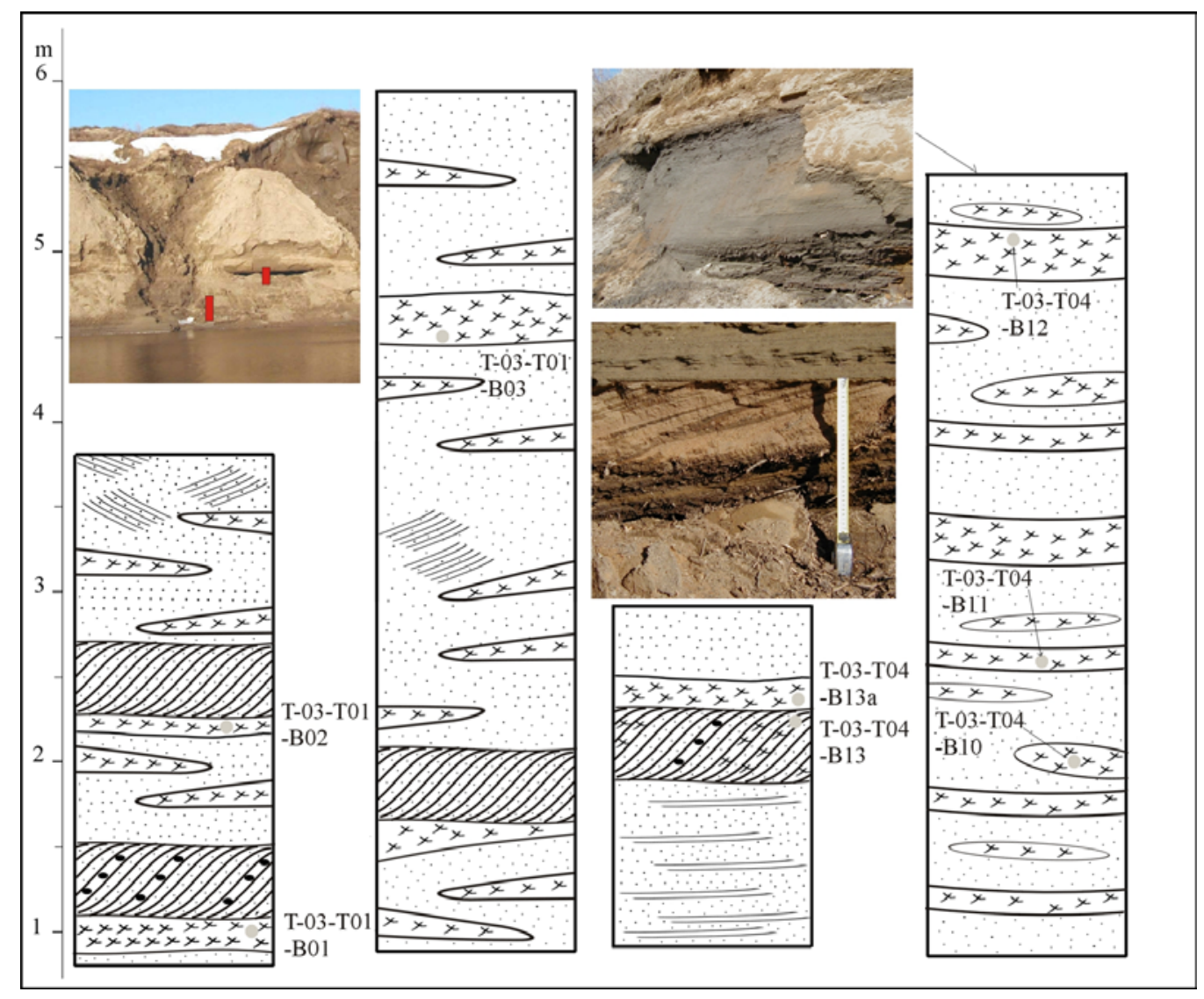

Fig. 4. Titaluk "Russian Section" (RS), profiles 1 (lower part) and 4.

Рис. 4. Разрез "Русский” (РС) на реке Титалук, расчистки 1 (низ) и 4.

iment. Yedoma-like sediments were observed in one profile only. This unit was inserted into the sand and probably filled an eroded depression. As we know from the well-developed Itkillik Yedoma deposit, the age of the ice- rich silt could range any time from 42 to $14 \mathrm{ka}$. We have to take into consideration that Yedoma deposits are quite rare on the Alaskan North Slope, in contrast to northeastern Siberia where they are widespread. On the North Slope, loess accumulation was local and was probably concurrent with more common sand dunes, and fluvial deposition.

Populus Beds were found in two profiles at RS (Figs 4, 5). These sediments are represented by fine sand and abundant plant layers consisting of well-preserved poplar leaves, small twigs, and wood pieces. Populus Beds filled eroded cuts inside the sandy unit. The age range of the Populus beds is $10-8 \mathrm{ka} \mathrm{yr}$. (Table 1). This interval includes two episodes of warming: preand post-Younger Dryas observed in both Eastern Beringia (Ager, 1982; Edwards, Barker, 1994; Mann et al., 2002, 2010) and Western Beringia (MacDonald et al., 2000; Smith et al., 2004; Grosse et al., 2007).

The second warming (around 9-8 ka), which corresponds with Boreal Period of the BlyttSernander scheme, was probably the warmest interval of the Holocene in Beringia (Kaufman et al., 2004). Trees spread north to coastal areas in Siberia (Kaplina, Lozhkin, 1982; Kuzmina, Sher, 2006), spruce forest reached the central Brooks Range (Anderson, Brubaker, 1994), and beaver 


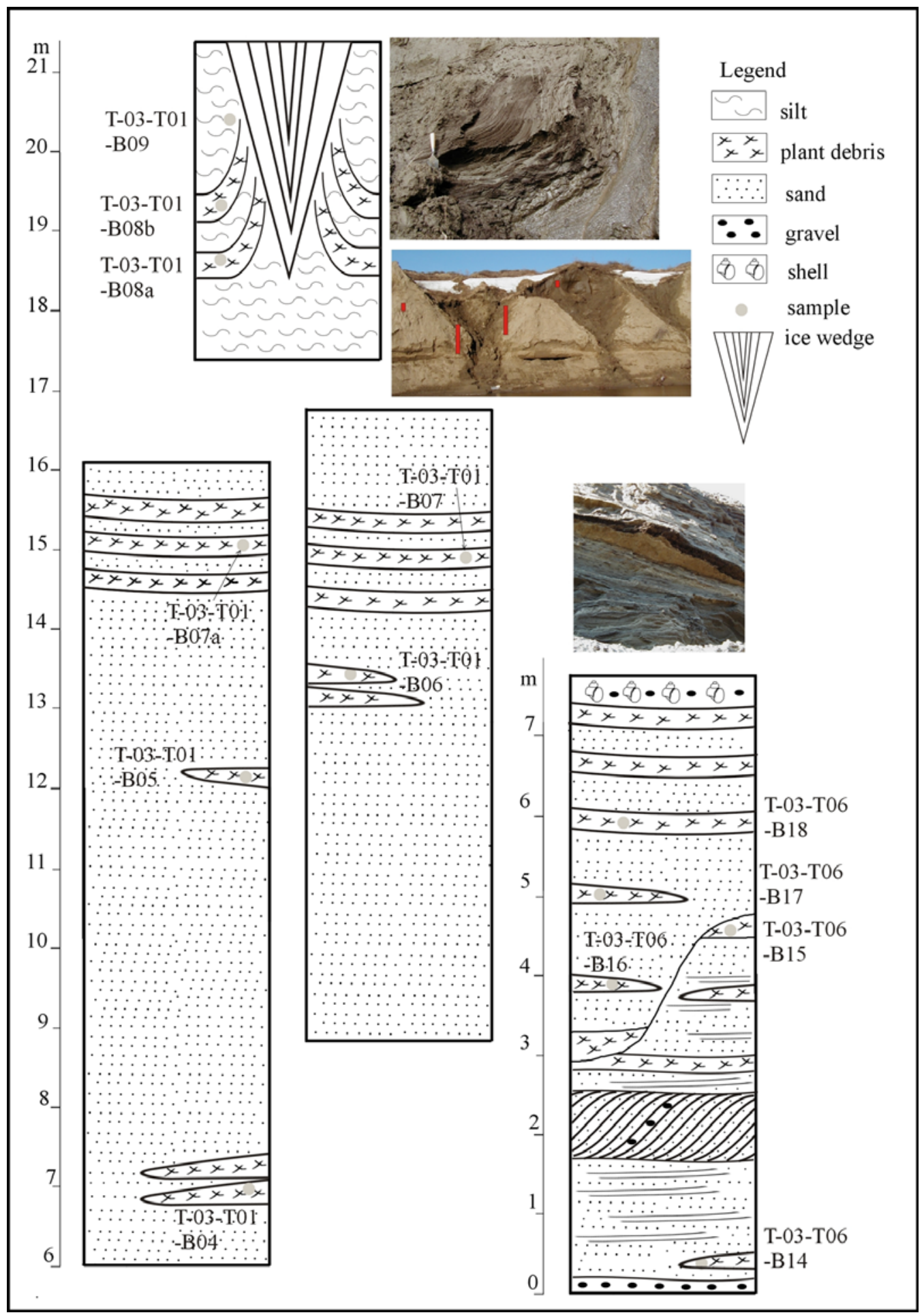

Fig. 5. Titaluk "Russian Section" (RS), profiles 1 (upper part) and 6.

Рис. 5. Разрез “Русский” (РС) на реке Титалук, расчистки 1 (верх) и 6. 


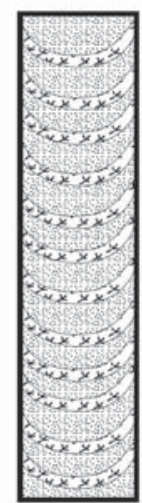

T03-B12

T03-B11

T03-B18

T03-B17

T03-B16

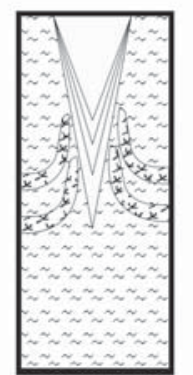

T03-B08b

T03-B08a
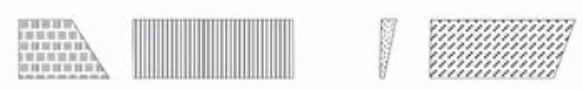

B - Sand, Late Pleistocene

T03-B07a

T03-B07

T03-B06

T03-B03

T03-B15
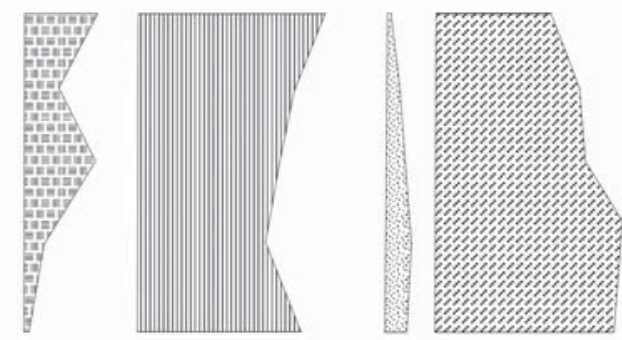

\section{A - Gravel, Pleistocene}

T03-B02

T03-B01

T03-B13a

T03-B13

T03-B14

IK-B05

IK-B04a

IK-B04

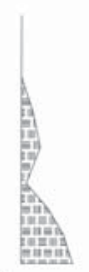

Steppe

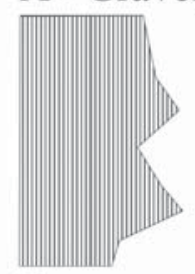

Dry tundra

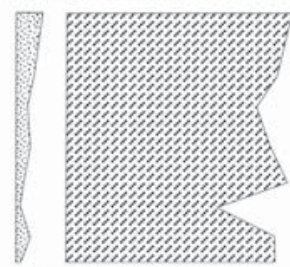

Arctic Mesic tundra

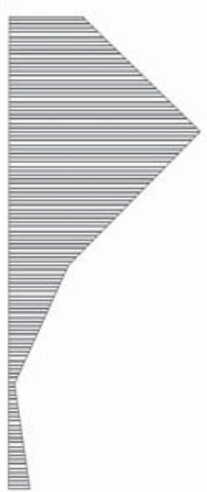

唈

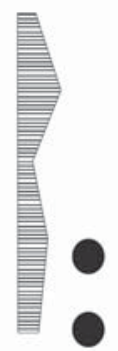

Forest

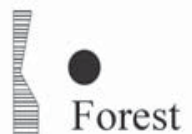

Aquatic, Riparian

Fig. 6. Scheme of ecological group variation in Ikpikpuk and Titaluk fossil insect assemblages.

Рис. 6. Схема изменений соотношения экологических групп в комплексах ископаемых насекомых с рек Икпикпук и Титалук. 
occupied formerly treeless landscapes in Alaska (Robinson et al., 2007). Climate on the North Slope was about 2 to $3^{\circ} \mathrm{C}$ warmer than today; and moisture was lower (Nelson, Carter, 1987).

\section{Material and Methods}

Insect samples were taken from 9 profiles (Figs 2-5), including 5 samples from the gravel unit at Ikpikpuk, 4 samples from the lower gravel unit similar to the lower unit at Titaluk, 7 samples from the main sandy unit at Titaluk, 3 samples from the Yedoma-analogue deposit, and 6 from the Populus Beds at Titaluk. The volume of each sample was different. Only 1-2 $\mathrm{kg}$ of sediment was sampled from the plant debris beds with visible insect remains; from less organic-rich deposits we took up to $50 \mathrm{~kg}$ per sample. Insects were concentrated by wet screening through $0.4 \mathrm{~mm}$ mesh and dried in the field, followed by later sieving of this concentrate in the laboratory, and picking of fossil insects with a binocular microscope (Sher, Kuzmina, 2007; Kuzmina et al., 2008).

Ecological interpretation of the fossil insect follows the previous studies (Matthews, 1983; Kuzmina et al., 2008, 2014). The method is based on the analysis of the proportion of ecological groups present in a particular sample.

Ecological groups (Table 2, Fig. 6) include:

1. st - indicators of Pleistocene steppetundra environments such as Aphodius pectoralis LeC. 1857, Morychus aff. aeneolus (LeC., 1863), Stephanocleonus confusus And., 1987, Coniocleonus parshus (And., 1987). These species live today in relict steppe communities in Alaska and the Yukon, or in xeric habitats to the south of this region.

The pill beetle Morychus aff. aeneolus has previously been recorded by other workers as Morychus sp. A, Morychus sp. B, or Morychus sp. (Matthews, 1968, 1983; Matthews, Telka, 1997). The taxonomic status of these very common Pleistocene beetles remains uncertain. We found a number of modern Morychus specimens on a relict steppe site near Kluane Lake, Yukon. These modern specimens are similar to the Pleistocene fossils in that they have a small elytral shoulder and shortened wings. At the same time the normal full wing M. aeneolus individuals are found on the Kluane steppe. The male genitalia of these two morphological types are slightly different. Since specimens of $M$. aeneolus are quite variable in shape, including shoulder size (Johnson, 1991), we can not confidently describe our specimens as a new species. All the Pleistocene specimens we found belong to the small shoulder morph and, if the species is indeed M. aeneolus, it is represented by stable (more than one million years) shortwing variation. So we decided to identify the Pleistocene beetles as M. aff. aeneolus (Kuzmina, Matthews, 2012; Murton et al., 2017).

Following the revision of Anderson (1987, 1989), Coniocleonus Motsch., 1860 and Stephanocleonus Motsch., 1860 are regarded here as subjective synonyms. Anderson (1987) examined type species of two genera and made a conclusion: "In view of this variation and the lack of other distinguishing characters, I cannot accept other than that Stephanocleonus is simply an apterous Coniocleonus. I do not think the rostral differences between the type species warrant separate generic status." (Anderson, 1987, p.453). "I choose to give the name Stephanocleonus priority over Coniocleonus because the former has been the only name used in reference to the Nearctic species here placed in the genus. Furthermore, no major works have recently appeared dealing with Coniocleonus, however, a recent review by Ter-Minasyan (1979) of the Stephanocleonus of the Palearctic Region includes species that in my opinion are congeneric with species in North America" (Anderson, 1987, p.454).

This decision was accepted (or partly accepted) by the international entomological community (for example Alonso-Zarazaga, 1999 noticed only Palearctic Coniocleonus) but was very inconvenient for environment reconstruction in Beringia. We can easily recognize the remains of two genera in the fossil assemblages. Coniocleonus sensu lato indicates meadowsteppe or dry tundra environments, but Stephanocleonus sensu stricto indicates thermophilous steppes. Fortunately, the genus Coniocleo- 


\begin{tabular}{|c|c|c|c|c|c|c|c|c|c|c|}
\hline \multirow{2}{*}{$\begin{array}{l}\text { sample } \\
\text { taxon }\end{array}$} & \multirow{2}{*}{$\begin{array}{l}\text { eco } \\
\text { code }\end{array}$} & \multicolumn{5}{|c|}{ Ikpikpuk B-0- } & \multicolumn{4}{|c|}{ Titaluk B-0- } \\
\hline & & 1 & 2 & 4 & $4 \mathrm{a}$ & 5 & 1 & 2 & 3 & 4 \\
\hline $\begin{array}{l}\text { Ord. Coleoptera } \\
\text { Fam. Carabidae } \\
\text { Subfam. Nebriinae }\end{array}$ & unit & A & A & A & A & A & A & A & B & $\mathrm{B}$ \\
\hline Notiophilus aquaticus (L., 1758) & $\mathrm{dt}$ & - & - & - & - & - & - & - & 1 & - \\
\hline $\begin{array}{l}\text { Notiophilus sp. } \\
\text { Subfam. Carabinae }\end{array}$ & $\mathrm{dt}$ & - & - & - & - & - & - & - & - & - \\
\hline Carabus truncaticollis Esch., 1833 & $\mathrm{mt}$ & - & - & - & - & - & - & - & - & - \\
\hline $\begin{array}{l}\text { Carabus sp. } \\
\text { Subfam. Elaphrinae }\end{array}$ & $\mathrm{mt}$ & - & - & - & 1 & - & 1 & - & 1 & - \\
\hline Elaphrus angusticollis Sahlb., 1844 & r\&a & - & - & - & - & - & - & - & - & - \\
\hline E. clairvillei Kby., 1837 & r\&a & - & - & - & - & - & - & - & - & - \\
\hline $\begin{array}{l}\text { Subfam. Scaritinae } \\
\text { Dyschirius nigricornis Motsch., } \\
1844\end{array}$ & $\mathrm{dt}$ & - & - & - & - & - & - & - & - & 1 \\
\hline Subfam. Trechinae & & & & & & & & & & \\
\hline Asaphidion alaskanum Wick., 1919 & r\&a & - & - & 1 & - & - & - & - & - & - \\
\hline A. yukonense Wick., 1919 & r\&a & - & - & - & 1 & - & - & - & - & - \\
\hline $\begin{array}{l}\text { Bembidion (Asioperyphus) } \\
\text { bimaculatum Kby., } 1837 \\
\text { B. (Asioperyphus) sordidum (Kby., }\end{array}$ & r\&a & - & - & - & 2 & 1 & 1 & - & - & - \\
\hline $\begin{array}{l}\text { 1837) } \\
\text { B. (Asioperyphus) umiatense } \\
\text { Lindr., } 1963 \\
\text { B. (Peryphanes) dauricum }\end{array}$ & $\begin{array}{l}\mathrm{r} \& \mathrm{a} \\
\mathrm{r} \& \mathrm{a}\end{array}$ & - & - & $\begin{array}{l}- \\
2\end{array}$ & $\begin{array}{l}- \\
2\end{array}$ & - & - & - & $\begin{array}{l}- \\
1\end{array}$ & - \\
\hline (Motsch., 1844) & $\mathrm{dt}$ & - & - & - & - & - & - & - & - & - \\
\hline $\begin{array}{l}\text { B. (Peryphanes) spp. } \\
\text { B. (Plataphus) hyperboraeorum } \\
\text { Mnst } 1923\end{array}$ & $\mathrm{dt}$ & - & - & $\begin{array}{l}- \\
1\end{array}$ & - & - & 1 & - & - & - \\
\hline $\begin{array}{l}\text { Mnst., } 1923 \\
\text { B. (Plataphodes) arcticum Lindr., } \\
1963\end{array}$ & $\begin{array}{l}\text { r\&a } \\
\text { r\&a }\end{array}$ & - & - & - & - & - & - & - & - & - \\
\hline $\begin{array}{l}\text { Bembidion sp. } \\
\text { Subfam. Harpalinae }\end{array}$ & r\&a & - & - & - & - & - & - & - & - & - \\
\hline $\begin{array}{l}\text { Tribe Harpalini } \\
\text { Dicheirotrichus mannerheimi } \\
\text { (Sahlb., 1844) }\end{array}$ & $\mathrm{dt}$ & - & - & - & 2 & - & - & - & - & - \\
\hline Harpalus amputatus Say, 1830 & $\mathrm{dt}$ & - & - & - & 1 & - & - & - & - & - \\
\hline H. vittatus alaskensis Lindt., 1968 & $\mathrm{dt}$ & - & - & - & 2 & 2 & - & - & - & - \\
\hline $\begin{array}{l}\text { Harpalus sp. } \\
\text { Tribe Lebiini }\end{array}$ & $\mathrm{dt}$ & - & - & - & - & - & 2 & - & - & - \\
\hline $\begin{array}{l}\text { Cymindis unicolor Kby., } 1837 \\
\text { Tribe Platynini }\end{array}$ & $\mathrm{dt}$ & - & - & - & - & - & - & - & - & - \\
\hline $\begin{array}{l}\text { Agonum (Europhilus) exaratum } \\
\text { (Mnnh., 1853) }\end{array}$ & r\&a & - & - & - & - & - & - & - & - & - \\
\hline
\end{tabular}


Table 2. List of insects from Ikpikpuk and Titaluk sections. Таблица 2. Список насекомых из разрезов Икпикпук и Титалук.

\begin{tabular}{ccccccccccccccccc}
\hline 5 & 6 & 7 & $7 \mathrm{a}$ & $8 \mathrm{a}$ & $8 \mathrm{~b}$ & 9 & 11 & $11 \mathrm{a}$ & 12 & 13 & $13 \mathrm{a}$ & 14 & 15 & 16 & 17 & 18 \\
\hline $\mathrm{B}$ & $\mathrm{B}$ & $\mathrm{B}$ & $\mathrm{B}$ & $\mathrm{C}$ & $\mathrm{C}$ & $\mathrm{C}$ & $\mathrm{D}$ & $\mathrm{D}$ & $\mathrm{D}$ & $\mathrm{A}$ & $\mathrm{A}$ & $\mathrm{A}$ & $\mathrm{B}$ & $\mathrm{D}$ & $\mathrm{D}$ & $\mathrm{D}$
\end{tabular}
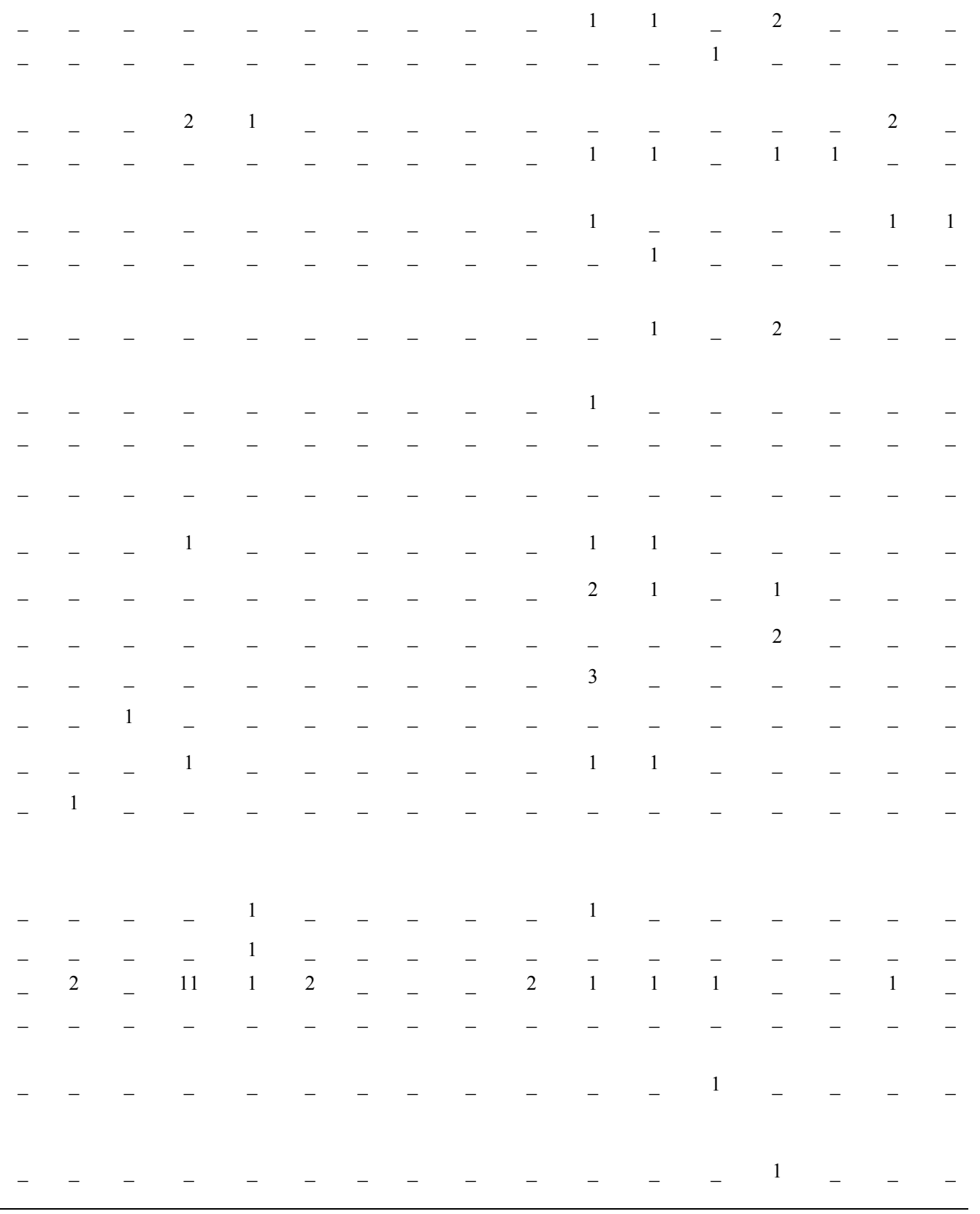


\begin{tabular}{|c|c|c|c|c|c|c|c|c|c|c|}
\hline \multirow{2}{*}{$\begin{array}{l}\text { sample } \\
\text { taxon }\end{array}$} & \multirow{2}{*}{$\begin{array}{l}\text { eco } \\
\text { code }\end{array}$} & \multicolumn{5}{|c|}{ Ikpikpuk B-0- } & \multicolumn{4}{|c|}{ Titaluk B-0- } \\
\hline & & 1 & 2 & 4 & $4 \mathrm{a}$ & 5 & 1 & 2 & 3 & 4 \\
\hline $\begin{array}{l}\text { Tribe Pterostichini } \\
\text { Poecilus (Derus) nearcticus Lindr., } \\
1966\end{array}$ & $\mathrm{dt}$ & - & 1 & 1 & 3 & 2 & - & - & 2 & - \\
\hline $\begin{array}{l}\text { Pterostichus (Cryobius) arcticola } \\
\text { Chad., } 1868\end{array}$ & & - & - & - & 7 & 18 & 14 & 14 & 7 & - \\
\hline $\begin{array}{l}\text { P. (Cryobius) brevicornis (Kby., } \\
\text { 1837) }\end{array}$ & $\mathrm{mt}$ & - & - & 18 & 21 & 29 & 5 & 5 & 31 & - \\
\hline P. (Cryobius) caribou Ball, 1962 & $\mathrm{mt}$ & - & - & 6 & - & 7 & 1 & - & 1 & - \\
\hline $\begin{array}{l}\text { P. (Cryobius) empetricola (Dej., } \\
\text { 1828) } \\
\text { P. (Cryobius) kotzebuei Ball, } 1962\end{array}$ & $\mathrm{mt}$ & - & - & 1 & - & - & - & - & - & - \\
\hline & $\mathrm{mt}$ & - & - & - & - & - & - & - & - & - \\
\hline P. (Cryobius) nivalis Sahlb., 1844 & $\mathrm{dt}$ & - & - & 29 & - & 1 & - & - & 5 & - \\
\hline $\begin{array}{l}\text { P. (Cryobius) parasimilis Ball, } \\
1962\end{array}$ & $\mathrm{mt}$ & - & - & - & - & 3 & 4 & 4 & 3 & - \\
\hline $\begin{array}{l}\text { P. (Cryobius) pinguedineus Esch., } \\
1823\end{array}$ & $\mathrm{mt}$ & - & 1 & 7 & 19 & 12 & 4 & 2 & 11 & 1 \\
\hline P. (Cryobius) pinguedineus group & $\mathrm{mt}$ & - & - & - & - & - & - & - & - & - \\
\hline P. (Cryobius) similis Mann., 1852 & $\mathrm{dt}$ & - & - & 6 & 17 & 23 & 13 & 7 & 11 & 1 \\
\hline P. (Cryobius) soperi Ball, 1966 & $\mathrm{mt}$ ? & - & - & - & - & - & 5 & - & - & - \\
\hline P. (Cryobius) tareumiut Ball, 1962 & $\mathrm{mt}$ & - & - & _- & 35 & 19 & 8 & 3 & 12 & 2 \\
\hline $\begin{array}{l}\text { P. (Cryobius) ventricosus Esch., } \\
1823\end{array}$ & $\mathrm{mt}$ & - & - & 9 & 12 & 28 & 4 & 3 & 7 & 1 \\
\hline P. (Cryobius) spp. & $\mathrm{mt}$ & 1 & - & 15 & - & - & 17 & 5 & 1 & 2 \\
\hline P. (Lenapterus) agonus Horn., 1880 & $\mathrm{mt}$ & - & - & 1 & - & - & - & - & - & - \\
\hline $\begin{array}{l}\text { P. (Lenapterus) costatus (Men., } \\
1851)\end{array}$ & $\mathrm{mt}$ & - & - & - & 2 & 1 & - & - & - & - \\
\hline $\begin{array}{l}\text { P. (Lenapterus) vermiculosus } \\
\text { (Men., 1851) } \\
\text { P. (Steropus) circulosus Lindr. }\end{array}$ & $\mathrm{mt}$ & - & - & - & 1 & - & - & - & - & - \\
\hline $1966 ?$ & $\mathrm{dt}$ & - & - & - & - & - & - & - & - & - \\
\hline $\begin{array}{l}\text { P. (Tundraphilus) sublaevis } \\
\text { (Sahlb., 1880) }\end{array}$ & $\mathrm{dt}$ & - & - & 2 & 5 & 7 & 1 & - & 2 & 1 \\
\hline $\begin{array}{l}\text { Stereocerus haematopus (Dej., } \\
\text { 1831) }\end{array}$ & $\mathrm{dt}$ & - & 1 & 1 & 7 & 4 & 4 & - & 2 & - \\
\hline $\begin{array}{l}\text { Tribe Zabrini } \\
\text { Amara (Amarocelia) erratica Duft., }\end{array}$ & & & & & & & & & & \\
\hline $\begin{array}{l}1812 \\
\text { A. (Amarocelia) interstitialis Dej., } \\
1828\end{array}$ & $\begin{array}{l}\mathrm{dt} \\
\mathrm{dt}\end{array}$ & - & - & $\begin{array}{l}- \\
1\end{array}$ & - & - & - & $\begin{array}{l}- \\
-\end{array}$ & $\begin{array}{l}- \\
-\end{array}$ & $\begin{array}{l}- \\
-\end{array}$ \\
\hline A. (Bradytus) glacialis Mnnh., 1853 & $\mathrm{dt}$ & - & - & - & - & - & - & - & - & - \\
\hline A. (Curtonotus) alpina Payk., 1790 & $\mathrm{dt}$ & - & - & 7 & 19 & 32 & 63 & 27 & 41 & 3 \\
\hline $\begin{array}{l}\text { A. (Curtonotus) bokori Csiki, } 1929 \\
\text { A. (Paracelia) quenseli Schnh., }\end{array}$ & $\mathrm{dt}$ & - & - & - & 1 & - & - & - & - & - \\
\hline 1806 & $\mathrm{dt}$ & - & - & - & - & - & - & - & - & - \\
\hline Fam. Dytiscidae & & & & & & & & & & \\
\hline Subfam. Agabinae & & & & & & & & & & \\
\hline Agabus confinis (Gyll., 1808)? & r\&a & - & - & - & - & - & - & - & - & - \\
\hline
\end{tabular}


Table 2 (continued). Таблица 2 (продолжение).

\begin{tabular}{ccccccccccccccccc}
\hline 5 & 6 & 7 & $7 \mathrm{a}$ & $8 \mathrm{a}$ & $8 \mathrm{~b}$ & 9 & 11 & $11 \mathrm{a}$ & 12 & 13 & $13 \mathrm{a}$ & 14 & 15 & 16 & 17 & 18 \\
\hline & 4 & 1 & 25 & 2 & 2 & - & 1 & 1 & 1 & 1 & 2 & - & 2 & - & 5 & 3 \\
- & 16 & 3 & 54 & 13 & 10 & - & 1 & 2 & 3 & 53 & 35 & 56 & 54 & 13 & 10 & 7 \\
- & 19 & 7 & 75 & 11 & 12 & 3 & - & 2 & 3 & 28 & 19 & 29 & 39 & 19 & 11 & 3 \\
- & - & - & 13 & 4 & - & - & - & - & - & 3 & 9 & 9 & 17 & 1 & - & - \\
- & - & - & 2 & - & - & - & - & - & - & - & - & - & - & - & - & - \\
- & 3 & - & - & - & - & - & - & - & - & - & - & - & - & - & - & - \\
- & - & - & 1 & - & - & - & - & - & - & - & - & - & - & - & - & - \\
- & 5 & - & 27 & - & - & - & - & - & 1 & - & - & 10 & 17 & 2 & 2 & - \\
- & 28 & 5 & 166 & 14 & 8 & 3 & 4 & 3 & 7 & 26 & 31 & 51 & 43 & 22 & 21 & 7 \\
- & - & - & 30 & 6 & 1 & - & - & - & - & 8 & 4 & - & 2 & - & - & - \\
- & 14 & - & 41 & 6 & 3 & - & - & - & - & 8 & 7 & 5 & 6 & 2 & 1 & 1 \\
- & - & - & 4 & - & - & - & - & - & - & - & - & 5 & 2 & - & 2 & - \\
- & 13 & 4 & 232 & 12 & 13 & 1 & - & 4 & 7 & 21 & 22 & 55 & 34 & 11 & 8 & 2 \\
- & 7 & - & 99 & 6 & 2 & - & 1 & - & 3 & 16 & 7 & 14 & 20 & 4 & 3 & - \\
- & 8 & 3 & 39 & - & - & - & 2 & - & 6 & 13 & 13 & 1 & 19 & 3 & 1 & - \\
- & - & - & 2 & - & - & - & - & - & - & 1 & - & - & 3 & - & - & - \\
- & - & - & 2 & - & - & - & - & - & 2 & - & - & - & - & 1 & 1 & - \\
- & - & 1 & - & - & - & - & - & - & - & - & 2 & - & 1 & - & - & - \\
- & - & - & - & - & - & - & - & - & - & 1 & - & - & - & - & - & - \\
- & 6 & - & 42 & 2 & 1 & 1 & 1 & - & 6 & 4 & 6 & 4 & 2 & 1 & 2 & 1 \\
- & 4 & - & 22 & 5 & 4 & - & 1 & - & 2 & 3 & 6 & 3 & 5 & 4 & 4 & 2 \\
- & & & & & & & & & & & & & & & & \\
-
\end{tabular}

$\begin{array}{ccccccccccccccccc}- & - & - & 1 & - & - & - & - & - & - & - & - & - & - & - & - & - \\ 1 & 65 & 25 & 652 & 55 & 37 & 3 & 2 & 1 & 21 & 66 & 54 & 154 & 137 & 50 & 48 & 32\end{array}$ 


\begin{tabular}{|c|c|c|c|c|c|c|c|c|c|c|}
\hline \multirow{2}{*}{$\begin{array}{l}\text { sample } \\
\text { taxon }\end{array}$} & \multirow{2}{*}{$\begin{array}{l}\text { eco } \\
\text { code }\end{array}$} & \multicolumn{5}{|c|}{ Ikpikpuk B-0- } & \multicolumn{4}{|c|}{ Titaluk B-0- } \\
\hline & & 1 & 2 & 4 & $4 \mathrm{a}$ & 5 & 1 & 2 & 3 & 4 \\
\hline A. moestus (Curt., 1835) & r\&a & - & - & - & - & 2 & 2 & 3 & 2 & - \\
\hline A. serricornis (Payk., 1799)? & r\&a & - & - & - & - & - & - & - & - & - \\
\hline A. thomsoni (Sahlb., 1871) & r\&a & - & - & - & - & - & - & - & - & - \\
\hline Agabus spp. & r\&a & - & - & - & - & - & - & - & - & - \\
\hline $\begin{array}{l}\text { Ilybius picipes (Kby., 1837) } \\
\text { Subfam. Colymbetinae }\end{array}$ & r\&a & - & - & - & - & - & - & - & - & - \\
\hline Colymbetes dahuricus Aub., 1837 & r\&a & - & - & - & - & - & - & - & - & - \\
\hline C. dolabratus (Payk., 1837) & r\&a & - & - & - & - & 1 & - & - & - & - \\
\hline $\begin{array}{l}\text { Colymbetes sp. } \\
\text { Subfam. Hydroporinae }\end{array}$ & r\&a & - & - & - & - & - & - & - & - & - \\
\hline $\begin{array}{l}\text { Hydroporus lapponum (Gyll., } \\
\text { 1808) }\end{array}$ & r\&a & - & - & - & - & - & - & - & - & - \\
\hline H. morio Aub., 1838 & r\&a & - & - & - & - & 1 & - & - & - & - \\
\hline H. polaris Fall, 1923 & r\&a & - & - & - & - & - & 2 & - & 1 & - \\
\hline H. striola (Gyll., 1826)? & r\&a & - & - & - & - & - & - & 1 & - & - \\
\hline Hydroporus spp. & r\&a & - & - & - & - & - & - & - & - & 1 \\
\hline Oreodytes laevis (Kby., 1837) & r\&a & - & - & - & - & - & - & - & - & - \\
\hline O. recticollis (Fall, 1926) & r\&a & - & - & - & - & - & - & - & - & - \\
\hline $\begin{array}{l}\text { O. sanmarkii (Sahl., 1826) } \\
\text { Fam. Hydrophilidae }\end{array}$ & r\&a & - & - & - & 1 & - & - & - & - & - \\
\hline $\begin{array}{l}\text { Subfam. Helophorinae } \\
\text { Helophorus sempervarians Ang., } \\
1970 ?\end{array}$ & r\&a & - & - & - & - & - & - & - & - & - \\
\hline H. splendidus Sahlb., 1880 & r\&a & - & - & - & 1 & - & 2 & - & 2 & - \\
\hline $\begin{array}{l}\text { Helophorus sp. } \\
\text { Subfam. Hydrophilinae }\end{array}$ & r\&a & - & - & - & - & - & - & - & - & - \\
\hline Cymbiodyta sp. & r\&a & - & - & - & - & - & - & - & - & - \\
\hline $\begin{array}{l}\text { Hydrobius fuscipes L., } 1758 \\
\text { Subfam. Sphaeridiinae }\end{array}$ & r\&a & - & - & - & - & - & - & - & - & - \\
\hline $\begin{array}{l}\text { Cercyon sp. } \\
\text { Fam. Leiodidae } \\
\text { Subfam. Leiodinae }\end{array}$ & $\mathrm{pl}$ & - & - & - & - & - & - & - & 1 & - \\
\hline Agathidium angulare Mann., 1852 & $\mathrm{pl}$ & - & - & - & - & - & - & - & - & - \\
\hline Hydnobius sp. & $\mathrm{pl}$ & - & - & - & - & - & - & - & - & - \\
\hline $\begin{array}{l}\text { Leiodes collaris (LeC., 1850) } \\
\text { Subfam. Cholevinae }\end{array}$ & $\mathrm{pl}$ & - & - & - & 1 & - & - & - & - & - \\
\hline $\begin{array}{l}\text { Catops basilaris Say, } 1823 ? \\
\text { Fam. Silphidae } \\
\text { Subfam. Silphinae }\end{array}$ & $\mathrm{pl}$ & - & - & - & - & - & - & - & 1 & - \\
\hline $\begin{array}{l}\text { Thanatophilus lapponicus (Hbst., } \\
\text { 1793) }\end{array}$ & & - & - & - & 1 & - & - & - & - & - \\
\hline
\end{tabular}


Table 2 (continued). Таблица 2 (продолжение).

\begin{tabular}{|c|c|c|c|c|c|c|c|c|c|c|c|c|c|c|c|c|}
\hline 5 & 6 & 7 & $7 \mathrm{a}$ & $8 \mathrm{a}$ & $8 \mathrm{~b}$ & 9 & 11 & $11 \mathrm{a}$ & 12 & 13 & $13 a$ & 14 & 15 & 16 & 17 & 18 \\
\hline - & 2 & - & - & - & - & - & - & 3 & - & - & 1 & - & 1 & 1 & 1 & - \\
\hline- & - & - & - & - & - & - & 8 & - & 4 & - & - & - & - & - & - & - \\
\hline- & - & - & 6 & - & 1 & - & - & - & - & - & - & - & - & - & - & - \\
\hline- & - & - & - & - & - & - & - & - & - & - & - & 5 & 1 & - & - & 1 \\
\hline- & - & - & - & - & - & - & - & - & - & - & 1 & - & - & - & - & - \\
\hline - & - & - & - & - & - & - & - & 3 & - & - & - & - & - & - & - & - \\
\hline- & - & - & 1 & 1 & - & - & - & - & - & 1 & 2 & - & - & - & - & 2 \\
\hline- & - & - & - & - & - & - & - & - & - & 1 & - & - & - & - & - & - \\
\hline- & - & - & 1 & - & 2 & - & - & - & - & 1 & - & - & - & - & - & - \\
\hline- & - & - & - & - & - & - & - & - & - & - & - & - & - & - & - & - \\
\hline _- & - & 2 & 3 & 1 & - & - & - & 2 & - & 3 & 4 & 1 & 4 & - & 1 & - \\
\hline- & - & - & - & - & - & - & - & - & - & - & - & - & - & - & - & - \\
\hline- & - & - & - & - & - & - & 1 & & - & - & - & 2 & - & - & - & - \\
\hline- & - & - & - & - & - & - & - & - & 1 & - & - & - & - & - & - & - \\
\hline- & - & - & - & - & - & - & - & - & - & - & 1 & - & - & - & - & - \\
\hline- & - & - & - & - & - & - & - & - & - & - & - & - & - & - & - & - \\
\hline- & - & - & - & - & - & - & - & - & - & - & - & - & 1 & - & - & - \\
\hline - & 1 & 1 & 2 & 1 & 1 & - & - & 1 & 1 & 2 & - & 2 & 1 & - & - & - \\
\hline- & - & - & - & - & - & - & - & - & - & - & 1 & - & - & - & 1 & - \\
\hline- & - & - & - & - & - & - & - & - & - & - & - & - & 1 & - & - & - \\
\hline- & - & - & - & - & - & - & - & - & - & - & - & 1 & 1 & - & - & - \\
\hline- & - & - & - & - & - & - & - & - & - & - & - & - & - & - & - & - \\
\hline- & - & - & - & - & - & - & - & - & - & 1 & 1 & 1 & 2 & - & - & - \\
\hline- & - & - & - & - & - & - & - & - & - & - & - & - & - & - & 1 & - \\
\hline - & 1 & - & 1 & _ & 1 & - & - & _ & - & - & 1 & - & - & - & _ & - \\
\hline
\end{tabular}




\begin{tabular}{|c|c|c|c|c|c|c|c|c|c|c|}
\hline \multirow{2}{*}{$\begin{array}{l}\text { sample } \\
\text { taxon }\end{array}$} & \multirow{2}{*}{$\begin{array}{l}\text { eco } \\
\text { code }\end{array}$} & \multicolumn{5}{|c|}{ Ikpikpuk B-0- } & \multicolumn{4}{|c|}{ Titaluk B-0- } \\
\hline & & 1 & 2 & 4 & $4 \mathrm{a}$ & 5 & 1 & 2 & 3 & 4 \\
\hline Th. sagax (Mann., 1853) & & _- & _ & _ & _- & _- & _- & _- & _- & _ \\
\hline Fam. Staphylinidae & & & & & & & & & & \\
\hline Subfam. Omaliinae & & & & & & & & & & \\
\hline Tribe Anthophagini & & & & & & & & & & \\
\hline Acidota quadrata (Zett., 1840) & $\mathrm{pl}$ & - & - & - & - & - & - & - & - & - \\
\hline Eucnecosum sp.? & $\mathrm{pl}$ & - & - & - & - & - & 1 & - & - & - \\
\hline Olophrum latum Maekl., 1853 & $\mathrm{r} \& \mathrm{a}$ & - & - & - & - & - & - & - & - & - \\
\hline O. rotundicolle (Sahlb., 1830) & $\mathrm{r} \& \mathrm{a}$ & - & - & - & - & - & - & - & - & 1 \\
\hline $\begin{array}{l}\text { Olophrum sp. } \\
\text { Tribe Omaliini }\end{array}$ & $\mathrm{r} \& \mathrm{a}$ & - & - & 1 & - & - & - & - & - & - \\
\hline $\begin{array}{l}\text { Micralymma brevilingue Schtd., } \\
1845\end{array}$ & $\mathrm{dt}$ & - & - & - & - & - & 2 & 1 & 1 & - \\
\hline Subfam. Tachyporinae & & & & & & & & & & \\
\hline Tribe Tachiporini & & & & & & & & & & \\
\hline Tachinus apterus Maekl., 1853 & ar & - & - & - & - & - & - & - & - & - \\
\hline T. brevipennis Sahlb., 1880 & $\mathrm{mt}$ & - & - & - & 4 & - & 35 & 11 & 5 & 5 \\
\hline T. instabilis Maekl., 1853 & $\mathrm{pl}$ & - & - & - & - & - & - & - & 1 & - \\
\hline Subfam. Steninae & & & & & & & & & & \\
\hline Stenus sp. & $\mathrm{r} \& \mathrm{a}$ & - & - & - & 1 & - & - & - & - & - \\
\hline Subfam. Paederinae & & & & & & & & & & \\
\hline Lathrobium nigrum LeC., 863 & $\mathrm{pl}$ & - & - & - & - & - & - & - & - & - \\
\hline L. sibiricum Fauvel, 1875 & $\mathrm{pl}$ & - & - & - & - & - & - & - & 1 & - \\
\hline L. washingtoni Csy., 1905 & $\mathrm{pl}$ & - & - & - & 2 & - & 1 & - & - & - \\
\hline Subfam. Staphylininae & & & & & & & & & & \\
\hline $\begin{array}{l}\text { Philonthus subvirescens Thom., } \\
1884\end{array}$ & $\mathrm{pl}$ & - & - & - & - & - & - & - & - & - \\
\hline Quedius fellmani (Zett., 1838) & $\mathrm{pl}$ & - & - & - & - & - & - & - & - & - \\
\hline Q. sublimbatus Maek1., $1853 ?$ & $\mathrm{pl}$ & - & - & - & - & - & - & - & - & 1 \\
\hline Quedius sp. & $\mathrm{pl}$ & - & - & - & 1 & - & - & - & - & - \\
\hline Fam. Scarabaeidae & & & & & & & & & & \\
\hline Aphodius pectoralis LeC., $1857 ?$ & st & - & - & - & - & - & - & - & - & - \\
\hline Aphodius sp. & $\mathrm{pl}$ & - & - & - & - & - & - & - & - & - \\
\hline Fam. Byrrhidae & & & & & & & & & & \\
\hline Subfam. Byrrhinae & & & & & & & & & & \\
\hline $\begin{array}{l}\text { Morychus aff. aeneolus (LeC., } \\
\text { 1863) }\end{array}$ & st & - & - & 10 & 29 & 16 & - & - & 5 & 2 \\
\hline Simplocaria sp. & $\mathrm{dt}$ & - & - & - & - & - & - & - & - & - \\
\hline Subfam. Syncalyptinae & & & & & & & & & & \\
\hline $\begin{array}{l}\text { Curimopsis albonotata (LeC., } \\
1861)\end{array}$ & $\mathrm{dt}$ & - & - & - & 1 & 3 & 1 & - & - & - \\
\hline C. echinata (LeC., 1850) & $\mathrm{dt}$ & - & - & 1 & - & - & - & - & - & 1 \\
\hline C. moosilauke Johns., 1986 & $\mathrm{dt}$ & - & - & - & 2 & - & - & _- & 1 & - \\
\hline
\end{tabular}


Table 2 (continued). Таблица 2 (продолжение).

\begin{tabular}{|c|c|c|c|c|c|c|c|c|c|c|c|c|c|c|c|c|}
\hline 5 & 6 & 7 & $7 \mathrm{a}$ & $8 \mathrm{a}$ & $8 \mathrm{~b}$ & 9 & 11 & $11 \mathrm{a}$ & 12 & 13 & $13 a$ & 14 & 15 & 16 & 17 & 18 \\
\hline- & - & - & - & - & - & - & - & - & - & - & - & 1 & 1 & - & - & $\overline{-}$ \\
\hline- & - & - & - & - & - & - & - & - & - & - & - & 1 & - & - & - & - \\
\hline- & - & - & - & - & - & - & - & - & - & - & - & - & - & - & - & - \\
\hline- & - & - & - & - & - & - & - & - & - & - & - & - & - & - & - & 1 \\
\hline- & - & - & - & - & - & - & - & - & - & - & 3 & - & 3 & - & 1 & - \\
\hline- & - & - & - & - & - & - & - & - & - & 1 & - & - & 1 & - & - & - \\
\hline- & 1 & 3 & 2 & 2 & - & - & 1 & - & - & - & - & - & 2 & - & - & - \\
\hline- & - & - & - & - & - & - & - & - & - & 1 & - & - & - & - & - & - \\
\hline - & 14 & 9 & 14 & 8 & 9 & 1 & 1 & 1 & 3 & 8 & 4 & 38 & 20 & - & 5 & 1 \\
\hline- & - & - & - & - & - & - & - & - & - & - & - & - & - & - & - & - \\
\hline- & - & - & - & - & - & - & - & - & - & - & - & 1 & - & - & - & - \\
\hline- & - & - & - & - & - & - & - & - & - & - & 2 & 1 & - & - & - & - \\
\hline- & 1 & - & - & 1 & - & - & - & - & - & 1 & - & - & 1 & - & - & - \\
\hline- & - & - & - & - & - & - & - & - & - & - & - & - & - & - & - & - \\
\hline- & - & - & - & - & - & - & - & - & 1 & 1 & - & - & - & - & - & 1 \\
\hline- & - & - & - & - & - & - & - & - & - & - & - & - & 1 & - & - & - \\
\hline- & - & - & - & - & - & - & - & - & - & - & - & - & - & - & - & - \\
\hline- & - & - & - & - & - & - & - & - & - & 1 & - & - & - & - & - & - \\
\hline- & - & - & 2 & - & - & - & - & - & - & 2 & - & - & - & - & - & - \\
\hline - & - & - & - & - & - & - & - & - & - & - & - & - & - & - & - & 4 \\
\hline
\end{tabular}

\begin{tabular}{|c|c|c|c|c|c|c|c|c|c|c|c|c|c|c|c|}
\hline - & 58 & 7 & 476 & 56 & 19 & 6 & 3 & 5 & 6 & 12 & 6 & 2 & 1 & 2 & 41 \\
\hline 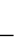 & - & - & - & - & - & - & - & - & - & - & - & - & 1 & - & - \\
\hline 1 & 4 & - & 9 & 1 & 2 & - & - & - & - & - & - & 3 & 3 & - & 3 \\
\hline - & - & - & - & - & - & - & - & - & - & - & - & - & - & - & - \\
\hline & _ & - & - & - & - & - & - & - & - & 2 & 4 & _ & - & _- & - \\
\hline
\end{tabular}




\begin{tabular}{|c|c|c|c|c|c|c|c|c|c|c|}
\hline \multirow{2}{*}{$\begin{array}{l}\text { sample } \\
\text { taxon }\end{array}$} & \multirow{2}{*}{$\begin{array}{l}\text { eco } \\
\text { code }\end{array}$} & \multicolumn{5}{|c|}{ Ikpikpuk B-0- } & \multicolumn{4}{|c|}{ Titaluk B-0- } \\
\hline & & 1 & 2 & 4 & $4 \mathrm{a}$ & 5 & 1 & 2 & 3 & 4 \\
\hline Curimopsis sp. & $\mathrm{dt}$ & - & - & - & - & - & - & - & - & - \\
\hline Fam. Coccinellidae & & & & & & & & & & \\
\hline Subfam. Coccinellinae & & & & & & & & & & \\
\hline Tribe Cephaloscymnini & & & & & & & & & & \\
\hline Ceratomegilla ulkei Crotch, 1873 & oth & - & - & - & - & - & - & - & - & - \\
\hline Tribe Coccinellini & & & & & & & & & & \\
\hline Coccinella fulgida Wats., 1954 & oth & - & - & - & - & - & - & - & - & 1 \\
\hline C. transversoguttata Fald., 1835 & oth & - & - & - & - & 1 & - & - & 1 & - \\
\hline Coccinella sp. & oth & - & - & - & 1 & - & - & - & - & - \\
\hline Fam. Chrysomelidae & & & & & & & & & & \\
\hline Subfam. Chrysomelinae & & & & & & & & & & \\
\hline $\begin{array}{l}\text { Chrysolina (Arctolina) magniceps } \\
\text { (Sahlb., 1887)? }\end{array}$ & ar & - & - & - & - & - & - & - & - & - \\
\hline $\begin{array}{l}\text { Ch. (Arctolina) septentrionalis } \\
\text { (Men, 1851) }\end{array}$ & $\mathrm{mt}$ & - & - & 1 & 3 & 5 & 6 & 2 & 1 & - \\
\hline $\begin{array}{l}\text { Ch. (Arctolina) subsulcata (Mnnh., } \\
\text { 1853) }\end{array}$ & ar & - & - & - & - & 2 & - & - & - & - \\
\hline $\begin{array}{l}\text { Ch. (Chalcoidea) marginata (L, } \\
\text { 1758) }\end{array}$ & $\mathrm{mt}$ ? & - & - & - & - & - & - & - & - & - \\
\hline Ch. (Pleurosticha) tolli Jac., 1990 & ar & - & - & - & - & - & 3 & 5 & 2 & - \\
\hline $\begin{array}{l}\text { Ch. (Allohypericia) basilaris (Say, } \\
\text { 1824) }\end{array}$ & $\mathrm{mt}$ ? & - & - & - & 1 & - & - & - & - & - \\
\hline Chrysolina sp. & $\mathrm{mt}$ ? & - & - & - & - & - & - & - & - & - \\
\hline Chrysomela blaisdelli (V.D., 1938) & $\mathrm{sh}$ & - & - & - & - & - & - & - & - & - \\
\hline Phratora kenaiensis Brown, 1952 & $\mathrm{sh}$ & - & - & - & - & - & - & - & - & - \\
\hline Phaedon armoraciae (L., 1758)? & $\mathrm{mt}$ & - & - & - & - & - & - & - & - & - \\
\hline Subfam. Galerucinae & & & & & & & & & & \\
\hline Tribe Galerucini & & & & & & & & & & \\
\hline $\begin{array}{l}\text { Galerucella stefanssoni (Brown, } \\
\text { 1938) }\end{array}$ & $\mathrm{mt}$ & - & - & - & - & 2 & - & - & - & - \\
\hline Fam. Brentidae & & & & & & & & & & \\
\hline Subfam. Apioninae & & & & & & & & & & \\
\hline $\begin{array}{l}\text { Mesotrichapion alaskanum (Fall, } \\
\text { 1926) }\end{array}$ & $\mathrm{dt}$ & - & - & 1 & - & - & - & - & 1 & - \\
\hline M. cyanitinctum (Fall, 1926) & $\mathrm{dt}$ & 1 & - & 1 & 9 & 1 & 2 & 1 & 4 & - \\
\hline Apioninae gen. indet. & $\mathrm{dt}$ ? & - & - & - & - & - & - & - & 1 & - \\
\hline Fam. Brachyceridae & & & & & & & & & & \\
\hline Subfam. Erirhininae & & & & & & & & & & \\
\hline Erirhinus aethiops (F., 1792) & $\mathrm{mt}$ & - & - & - & - & 2 & - & - & - & - \\
\hline Fam. Curculionidae & & & & & & & & & & \\
\hline Subfam. Ceutorhynchinae & & & & & & & & & & \\
\hline $\begin{array}{l}\text { Ceutorhynchus subpubescens LeC., } \\
1876\end{array}$ & st & - & - & - & - & - & - & - & - & - \\
\hline
\end{tabular}


Table 2 (continued). Таблица 2 (продолжение).

\begin{tabular}{|c|c|c|c|c|c|c|c|c|c|c|c|c|c|c|c|c|}
\hline 5 & 6 & 7 & $7 a$ & $8 \mathrm{a}$ & $8 b$ & 9 & 11 & $11 \mathrm{a}$ & 12 & 13 & $13 a$ & 14 & 15 & 16 & 17 & 18 \\
\hline- & - & - & - & - & - & - & 1 & 1 & - & - & - & - & - & - & - & - \\
\hline
\end{tabular}

$\begin{array}{lllllllllllllllll}- & 6 & 2 & 112 & 5 & 3 & 1 & - & 1 & 2 & 16 & 10 & 16 & 15 & 7 & 2 & - \\ - & - & - & 7 & - & - & - & - & - & 1 & - & - & - & 10 & 7 & - & - \\ - & - & - & 10 & 1 & - & - & - & - & - & - & 7 & 1 & 14 & 3 & 3 & 2 \\ - & - & - & - & - & - & - & - & - & - & - & - & - & - & - & - & - \\ - & 2 & - & 20 & 1 & - & - & - & 1 & - & 3 & 1 & - & 1 & 2 & 2 & - \\ - & - & - & - & - & - & - & 1 & - & - & - & - & - & - & - & - & - \\ - & - & - & 2 & - & - & - & - & - & 6 & - & 2 & - & - & - & 1 & - \\ - & - & - & - & - & - & - & - & - & - & 1 & - & - & - & - & - & - \\ - & - & - & - & - & - & - & - & - & - & - & - & 1 & - & - & - & -\end{array}$

$\begin{array}{lllllllllllllllll}- & 4 & - & 10 & 3 & 2 & - & - & 1 & - & 5 & 3 & 1 & 4 & - & 1 & - \\ - & 8 & 2 & 6 & 1 & 2 & 1 & - & 1 & - & - & 7 & 2 & 2 & - & 4 & 2 \\ - & - & - & - & - & - & - & - & - & - & - & - & - & - & - & - & -\end{array}$

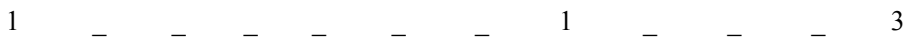




\begin{tabular}{|c|c|c|c|c|c|c|c|c|c|c|}
\hline \multirow{2}{*}{$\begin{array}{l}\text { sample } \\
\text { taxon }\end{array}$} & \multirow{2}{*}{$\begin{array}{l}\text { eco } \\
\text { code }\end{array}$} & \multicolumn{5}{|c|}{ Ikpikpuk B-0- } & \multicolumn{4}{|c|}{ Titaluk B-0- } \\
\hline & & 1 & 2 & 4 & $4 \mathrm{a}$ & 5 & 1 & 2 & 3 & 4 \\
\hline \multicolumn{11}{|l|}{ Subfam. Entiminae } \\
\hline \multicolumn{11}{|l|}{ Tribe Alophinini } \\
\hline $\begin{array}{l}\text { Lepidophorus lineaticollis Kby., } \\
1837\end{array}$ & $\mathrm{dt}$ & - & - & 8 & 16 & 93 & 3 & 2 & 12 & 2 \\
\hline L. thulius (Kiss., 1974) & $\mathrm{dt}$ & - & - & - & 1 & 3 & - & - & - & - \\
\hline \multicolumn{11}{|l|}{ Tribe Sitonini } \\
\hline Sitona aquilonius Bright, 1994 & dt & - & - & - & 1 & 7 & - & - & - & - \\
\hline S. lineellus (Bonsd., 1785) & $\mathrm{dt}$ & - & - & - & - & 1 & - & - & - & - \\
\hline \multicolumn{11}{|l|}{ Subfam. Hyperinae } \\
\hline Hypera castor (LeC., 1876) & $\mathrm{dt}$ & - & - & - & - & - & - & - & - & - \\
\hline H. diversipunctata Schrank, 1798 & $\mathrm{dt}$ & - & - & 1 & - & 2 & - & - & - & - \\
\hline H. trivittata (Say., 1831) & $\mathrm{dt}$ & - & - & - & 2 & 1 & - & - & - & - \\
\hline Hypera sp. & $\mathrm{dt}$ & - & - & - & - & - & - & - & - & - \\
\hline \multicolumn{11}{|l|}{ Subfam. Lixinae } \\
\hline \multicolumn{11}{|l|}{ Tribe Cleonini } \\
\hline $\begin{array}{l}\text { Stephanocleonus confusus (And., } \\
\text { 1987) }\end{array}$ & st & - & - & - & - & - & - & - & - & - \\
\hline Coniocleonus parshus (And., 1987) & st & - & - & - & - & - & - & - & - & - \\
\hline C. zherichini T.-M. et Kor., 1977 & $\mathrm{dt}$ & - & - & 3 & - & 9 & - & - & 4 & - \\
\hline Coniocleonus sp. & st? & - & - & - & - & - & - & - & - & - \\
\hline \multicolumn{11}{|l|}{ Subfam. Molytinae } \\
\hline \multicolumn{11}{|l|}{ Tribe Hylobiini } \\
\hline $\begin{array}{l}\text { Hylobius pinicola (Coup., } 1864 \text { ) } \\
\text { Tribe Lepyrini }\end{array}$ & & - & \multicolumn{2}{|c|}{ Tribe Lepyrini } & - & - & - & - & 1 & - \\
\hline Lepyrus canadensis Csy., 1895 & sh & - & - & - & - & 4 & - & - & - & - \\
\hline L. gemellus Kby., 1837 & sh & - & - & 1 & - & 3 & - & - & - & - \\
\hline L. nordenskioeldi Faust, 1885 & sh & - & - & 2 & 2 & 22 & 1 & - & 2 & - \\
\hline L. labradorensis Blair, 1933? & sh & - & - & - & - & - & - & - & - & - \\
\hline Lepyrus spp. & sh & - & - & - & - & - & - & - & - & - \\
\hline \multicolumn{11}{|l|}{ Subfam. Curculioninae } \\
\hline \multicolumn{11}{|l|}{ Tribe Rhamphini } \\
\hline Isochnus arcticus Kor., 1977 & ar & 2 & - & 1 & 6 & 1 & 7 & 2 & 12 & 1 \\
\hline \multicolumn{11}{|l|}{ Subfam. Scolytinae } \\
\hline \multicolumn{11}{|l|}{ Tribe Halastini } \\
\hline $\begin{array}{l}\text { Scierus annectans LeC., } 1876 \\
\text { Tribe Scolytini }\end{array}$ & fo & - & - & - & - & - & - & - & - & - \\
\hline Scolytus piceae (Swaine, 1910) & fo & - & - & - & - & - & 1 & - & - & - \\
\hline Ord. Heteroptera & & & & & & & & & & \\
\hline Fam. Corixidae & & & & & & & & & & \\
\hline Sigara sp. & $\mathrm{r} \& \mathrm{a}$ & - & - & - & - & - & 1 & - & - & - \\
\hline
\end{tabular}


Table 2 (continued).

Таблица 2 (продолжение).

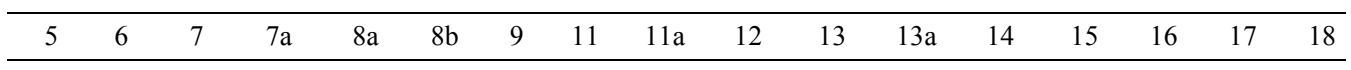

$\begin{array}{ccccccccccccccccc}1 & 26 & 6 & 699 & 33 & 12 & 7 & 4 & 4 & 6 & 26 & 40 & 7 & 85 & 18 & 47 & 65 \\ - & - & 1 & 8 & 3 & 2 & - & - & - & - & - & - & - & 2 & - & 1 & 1 \\ - & 2 & - & 9 & 2 & 2 & - & - & - & 1 & 4 & 3 & - & 1 & - & 1 & 4 \\ - & - & - & - & - & - & - & - & - & - & - & - & - & - & - & 1 & - \\ & & & & & & & & & & & & & & & & \\ - & - & - & - & - & - & - & - & - & - & 1 & - & - & - & - & - & - \\ - & 1 & - & 2 & 1 & - & - & - & - & - & - & 1 & - & 1 & - & 1 & - \\ - & - & - & - & - & - & - & - & - & - & - & - & - & - & - & - & - \\ - & - & - & - & - & 1 & - & - & - & - & - & - & - & - & - & - & - \\ & & & & & & & & & & & & & & & & \\ & & & & & & & & & & & & & & & & \\ - & - & - & - & 5 & 1 & - & - & - & - & 2 & 3 & - & 3 & - & - & - \\ - & - & - & 11 & - & - & - & - & - & - & - & - & - & 1 & - & - & 16 \\ - & 2 & 1 & 8 & - & - & - & - & 1 & 2 & - & - & 1 & - & 3 & 4 & 7 \\ - & - & - & 49 & - & - & - & - & - & - & - & - & - & - & - & - & -\end{array}$

$\begin{array}{lllllllllllllllll}- & - & - & 12 & 1 & - & - & - & - & - & 2 & - & - & - & - & - & - \\ - & - & - & 6 & - & - & - & - & - & 1 & 2 & - & - & 3 & 2 & - & - \\ - & 5 & - & 69 & 3 & - & - & 1 & 3 & 3 & 11 & - & 4 & 7 & 4 & 4 & 25 \\ - & - & - & - & - & - & - & - & - & & 1 & - & - & - & - & - & - \\ - & - & 1 & - & - & 1 & 1 & - & - & - & - & - & - & - & - & - & -\end{array}$

$\begin{array}{llllllllllllllllll}- & 12 & 3 & 12 & 1 & 7 & \ldots & - & 1 & 1 & 11 & 9 & 8 & 25 & - & 3 & 1\end{array}$ 


\begin{tabular}{|c|c|c|c|c|c|c|c|c|c|c|}
\hline \multirow{2}{*}{$\begin{array}{l}\text { sample } \\
\text { taxon }\end{array}$} & \multirow{2}{*}{$\begin{array}{l}\text { eco } \\
\text { code }\end{array}$} & \multicolumn{5}{|c|}{ Ikpikpuk B-0- } & \multicolumn{4}{|c|}{ Titaluk B-0- } \\
\hline & & 1 & 2 & 4 & $4 a$ & 5 & 1 & 2 & 3 & 4 \\
\hline \multicolumn{11}{|l|}{ Fam. Saldidae } \\
\hline Saldula sp. & $\mathrm{r} \& \mathrm{a}$ & - & - & - & - & - & - & - & - & - \\
\hline Chiloxanthus sp. & r\&a & - & - & - & - & - & - & - & - & - \\
\hline \multicolumn{11}{|l|}{ Ord. Trichoptera } \\
\hline Trichoptera larvae & $\mathrm{r} \& \mathrm{a}$ & - & - & - & - & _- & _- & - & - & - \\
\hline sum & & 4 & 3 & 139 & 246 & 371 & 218 & 98 & 201 & 27 \\
\hline
\end{tabular}

nus is regarded as valid again (Meregalli, Fremuth, 2013; Meregalli, 2017) but confusion concerning North American species still exists. Recently (Meregalli, 2017) part of the North American Stephanocleonus has been returned to Coniocleonus: Stephanocleonus stenotho$\operatorname{rax}$ And., 1987 = Coniocleonus zherichini T.M. et Kor., 1977; Stephanocleonus parshus And., 1987 = Coniocleonus parshus (And., 1987); Stephanocleonus plumbeus LeC., 1876 = Coniocleonus plumbeus (LeC). Two North American Stephanocleonus: S. confusus And., 1987 and S. cristatus LeC., 1876 remained in this genus.

2. dt - insects of dry, open habitats in the tundra and partly in boreal forest: Lepidophorus lineaticollis, Coniocleonus zherichini, Sitona aquilonius Bright, 1994, Hypera diversipunctata (Schrank, 1798), Amara alpina, Stereocerus haematopus and others.

3. $\mathrm{mt}$ - insects associated with mesic to moist tundra habitats or meadows and bogs in boreal forest. These include Pterostichus (Cryobius) spp., Tachinus brevipennis Sahlb., 1880, Chrysolina septentrionalis (Men., 1851), and others.

4. ar - Arctic insects. There are cold-resistant beetles, living today in the northern tundra and polar desert: Chrysolina subsulcata (Mann., 1853), Ch. tolli (Jac., 1910), Isochnus arcticus Kor., 1977.

5. sh - insects that live and feed on shrubs, mostly willows: Lepyrus spp., Chrysomela spp. and others.

6. pl — plant litter inhabitants including many species of rove beetles (Lathrobium spp., Quedius spp.) and Leiodidae

7. fo - forest species. Here the group is represented by two species of bark beetles ( $\mathrm{Sci}$ erus annectans LeC., 1876, Scolytus piceae (Swaine, 1910)).

8. r\&a - riparian and aquatic insects (Elaphrus spp., Bembidion umiatense Lindr., 1963, Agabus moestus (Curt., 1835)

9. oth - other insects, without certain ecological status, such as lady birds Coccinella spp.

We calculated the minimum number of individuals (MNI), based on the maximum number of any individual sclerite (head, pronotum, elytron) of a taxon in a sample. All MNI of certain ecological group are summed up. The result is graphic of ecological change of the insect assemblages thought the section (Fig. 6).

\section{Results}

Fossil insects from the area have previously been studied by R. Nelson. He made his Ph.D. dissertation (Nelson, 1982, 1986) on a study of Pleistocene and Holocene fossil insects from sites on the Ikpikpuk and Titaluk Rivers. He presented his results in meetings (Nelson, Carter, 1997; Nelson, 1998) and published a detailed paper about the insects of the Populus Beds from the Ikpikpuk section called Cottonwood bend (Nelson, Carter, 1987). Until now, no further work on the fossil insects of the North Slope has been done, despite their abundance and good preservation. 
Table 2 (continued).

Таблица 2 (продолжение).

\begin{tabular}{|c|c|c|c|c|c|c|c|c|c|c|c|c|c|c|c|c|}
\hline 5 & 6 & 7 & $7 a$ & $8 \mathrm{a}$ & $8 b$ & 9 & 11 & $11 \mathrm{a}$ & 12 & 13 & $13 a$ & 14 & 15 & 16 & 17 & 18 \\
\hline- & - & 2 & - & - & 1 & - & - & - & - & - & - & - & - & - & - & - \\
\hline- & - & - & - & - & - & - & - & - & 1 & - & - & - & 2 & - & - & 1 \\
\hline 1 & - & - & - & - & - & - & - & - & - & - & - & - & - & - & - & - \\
\hline 4 & 346 & 92 & 3114 & 272 & 165 & 26 & 34 & 43 & 105 & 397 & 348 & 506 & 639 & 186 & 254 & 207 \\
\hline
\end{tabular}

The fossil insects from Titaluk and Ikpikpuk are presented in Table 2 and Figs 7, 8.

Insects from the lower gravel (A) come from both sites (Figs 3-6). Samples Ikpikpuk 1 and 2 are poor, sample 3 is empty, but samples $4,4 \mathrm{a}$ and 5 yielded good results. The lower unit was sampled from the banks of the Titaluk River in 3 profiles; all 5 samples are rich (up to 500 $\mathrm{MNI}$ ).

Tundra species are represented very well: there are several species of the typical tundra ground beetle subgenus Pterostichus (Cryobius), including $P$. brevicornis, $P$. ventricosus, P. pinguedineus, $P$. caribou, P. nivalis, $P$. tareumiut, and others. Abundance and diversity of Cryobius is observed in each studied samples.

Remains of other cold-adapted ground beetles include Amara (Curtonotus) alpina, Pterostichus (Tundraphilus) sublaevis, and Stereocerus haematopus. These taxa are abundant throughout the whole section.

The relict tundra ground beetle Poecilus (Derus) nearcticus was found here as well as in other North Slope assemblages. This species is common in fossil assemblages in both Eastern and Western Beringia. The species was originally described (Lindroth, 1966) from the Anderson River Delta. Later it was discovered in northeastern Siberia (Kiselev, 1981). We found modern specimens on a sandy slope with scarce vegetation at the Titaluk River. The modern distribution of $P$. nearcticus reflects its Beringian origin.

The most common beetle in the whole section is the weevil Lepidophorus lineaticollis.
This beetle is common in modern tundra, relict steppe and openings in the boreal forest in northern North America. Recently the species has been recorded from Chukotka (Berman et al., 2002); in the Pleistocene it lived only in Eastern Beringia (Kuzmina, Matthews, 2012). L. lineaticollis indicates dry tundra or steppe tundra environments (Matthews, 1983).

Xerophilous insects dominate only one assemblage, IK-B05 (49\%) in the lower unit; other assemblages yield up to $52 \%$ mesic tundra species, with the most numerous being specimens of Pterostichus (Cryobius) and the rove beetle Tachinus brevipennis. However, all the samples except T-B01 and T-B02 contain species associated with of steppe-tundra environments, especially Morychus aff. aeneolus. All assemblages yielded members of the arctic group, including the weevil Isochnus arcticus and the leaf beetle Chrysolina tolli. Curiously, sample T-B01 yielded a clear indicator of coniferous forest, the spruce bark beetle Scolytus piceae. Such a strange combination of arctic and forest beetles could have been caused by rapid changes of environment and accumulation of the beetle remains in an alluvial deposit. Part of the fossil assemblage was probably reworked but in any case the presence of forest beetle in this region suggests at least a short warming interval.

Quaternary deposits in the Ikpikpuk-Titaluk area contain tree remains; there are big willow stumps in growth position, as well as poplar stumps and leaves. Poplar remains were found mostly in Populus Beds, but several willow 


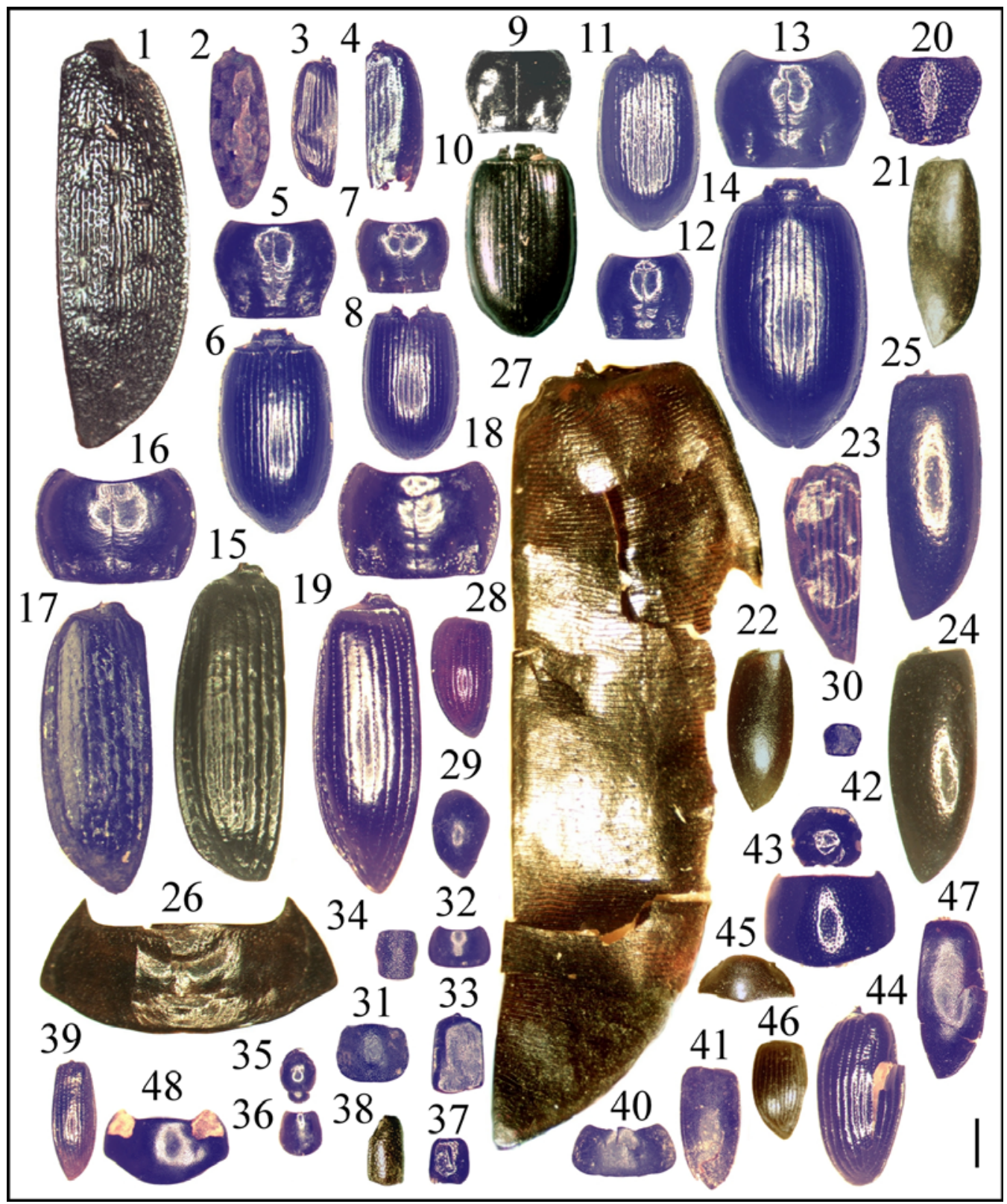

Fig. 7. Fossil insects (Carabidae - Coccinellidae) from the Titaluk river site.

1 - Carabus truncaticollis, 2 - Elaphrus parviceps, 3 - Bembidion arcticum, 4 - B. sordidum, 5, 6 - Pterostichus arcticola, 7, $8-P$. brevicornis, 9, $10-P$. empetricola $11-P$. pinguedineus, $12-P$. tareumiut, $13,14-P$. ventricosus, 15 - P. sublaevis, 16, 17 - Stereocerus haematopus, 18, 19-Amara alpina, 20 - Cymindis unicolor, 21 - Hydroporus lapponum, 22 - H. polaris, 23 - Oreodytes laevis, 24 - Agabus confinis, 25 - A. serricornis; 26 - Colymbetes dolabratus, 27 - C. dahuricus, 28 - Leiodes collaris, 29 - Agathidium angulare, 30 Micralymma brevilingue, 31 - Olophrum rotundicolle, 32 - Tachinus brevipennis, 33 - Tachinus sp., 34 - Stenus sp., 35, 36 - Philonthus subvirescens, 37 - Lathrobium sibiricum, 38 - Quedius sublimbatus, 39 - Helophorus splendidus, 40, 41 - Cymbiodyta sp., 42, 43, 44 - Aphodius pectoralis, 45, 46 - Curimopsis albonotata, 47 Ceratomegilla ulkei, 48 - Coccinella transversoguttata; 1 - T03-B17; 2, 3, 4, 5, 6, 7, 8, 11, 12, 13, 14, 17, 18, 19, 24, 26, 35, 36, 37 - Т03-B13; 9, 10, 21, 22 - T03-B07a; 16, 29, 31, 32, 33, 40, 41 - T03-B15; 20, 34- T03-В14; 23, 25 - T03-В12; 27 - T03-В11a; 28, 45, 46 - T03-В06; 30 - T03-В11; 38 - T03-В04; 39, 48 - T03-В03; 
stumps and a single poplar stump were found in the lower gravel (Mann et al., 2010). Spruce remains are rare there. A few fragmental spruce needles were observed in the Populus Beds (Nelson, Carter, 1987); they are poorly preserved, highly oxidized and are believed to be reworked. Our team found a spruce stump on a sandy bar on the Titaluk River. So, spruce grew in the area in some period of the Pleistocene, but this was an exceptional episode.

Reworking of fossil insects likely took place here. Usually erosion, flotation, coarse gravel and other impacts destroy fragile chitin fragments. But here we can see some evidence of possible reworking. Coarse alluvium of the lower unit contains well- preserved chitin and the concentration of beetle fragments is extremely high. These fragments were probably sorted by size, for example big and heavy elytra of $L e$ pyrus were concentrated in a cross-bedded layer with coarse gravel (sample T-B13) while they are absent in the sample T-B13a which was taken $10 \mathrm{~cm}$ higher, from sediments containing tiny plant debris. Local chitin preserved under permafrost conditions is not fragile.

Perhaps the repetitive ecological composition of the insect assemblages throughout the whole section (Fig. 6, Table 2) could be partly explained by reworking.

The proportion of riparian and aquatic insects is low in the gravel unit. Aquatic species are represented by the widespread arctic diving beetles Agabus moestus, Colymbetes dolabratus, various species of Hydroporus, and the water scavenger beetle Helophorus splendidus. These beetles prefer small vegetated standing water, not a fast flowing river. Probably they occupied pools in the river floodplain. The floodplain also provided habitats for Bembidi- on umiatense, Olophrum latum, O. rotundicolle, Stenus sp. and others.

The main sandy unit (B) was sampled in 4 profiles of the Titaluk section (Figs 4-6). The abundance of insect fossils varied greatly between samples (Table 2) from almost none to very rich. The richest sample (T-B07a) contained a minimum of 3114 individuals. The layer from which the samples were taken was full of insect remains; it was a very rare case in which insect chitin actually formed the bulk of the sediment.

Insect assemblages taken from the sand unit are more-or-less similar to those described above. However, there is evidence of increasing environmental aridity with age, because the proportion of dry tundra and steppe-tundra groups became more prominent in the upper samples. Weevils play a more significant role than before, especially xerophilous species such as Sitona aquilonius, Hypera diversipunctata, Stephanocleonus confusus, Coniocleonus parshus, and C. zherichini. These species are quite rare in the modern environment but were common in the Pleistocene. The most interesting discovery was the finding of S. confusus and C. parshus. The first species is known today from southern Alberta and Saskatchewan and south to Arizona; the second is widely distributed in North America as far north as northern Alberta and British Columbia, but does not reach Alaska. Neither species has been recorded from Arctic tundra. The third species, $C$. zherichini, has a trans-Beringian distribution today, but this species is quite rare in modern collections. These southern weevils, in combination with extremely abundant fossils of Lepidophorus lineaticollis, the exotic Morychus aff. aeneolus, and the relict Poecilus nearcti-

42, 43, 44, 47 - T03-B17; 1, 2, 4, 21, 22, 24, 25, 27, 28, 38, 39, 40, 44, 46, 47 — right elytron; 3, 15, 17, 19, 23, 29, 33 - left elytron; $6,8,10,14$ - both elytra; $5,7,9,11,12,13,16,18,20,26,30,31,34,37,40,43,45,48$ - pronotum; 35,42 - head. Scale bar $1 \mathrm{~mm}$.

Рис. 7. Ископаемые насекомые (Carabidae - Coccinellidae) из разреза на реке Титалук.

1 - T03-B17; 2, 3, 4, 5, 6, 7, 8, 11, 12, 13, 14, 17, 18, 19, 24, 26, 35, 36, 37 - T03-B13; 9, 10, 21, 22 - T03-B07a; 16, 29, 31, 32, 33, 40, 41 - T03-B15; 20, 34 - T03-B14; 23, 25 - T03-B12; 27 - T03-B11a; 28, 45, 46 - T03В06; 30 - Т03-В11; 38 - Т03-В04; 39, 48 - Т03-В03; 42, 43, 44, 47 - Т03-В17; 1, 2, 4, 21, 22, 24, 25, 27, 28, 38, 39, 40, 44, 46, 47 - правое надкрылье; 3, 15, 17, 19, 23, 29, 33 - левое надкрылье; 6, 8, 10, 14 - соединенные надкрылья; 5 , 7, 9, 11, 12, 13, 16, 18, 20, 26, 30, 31, 34, 37, 40, 43, 45, 48 - переднеспинка; 35, 42 — голова. Шкала 1 мм. 


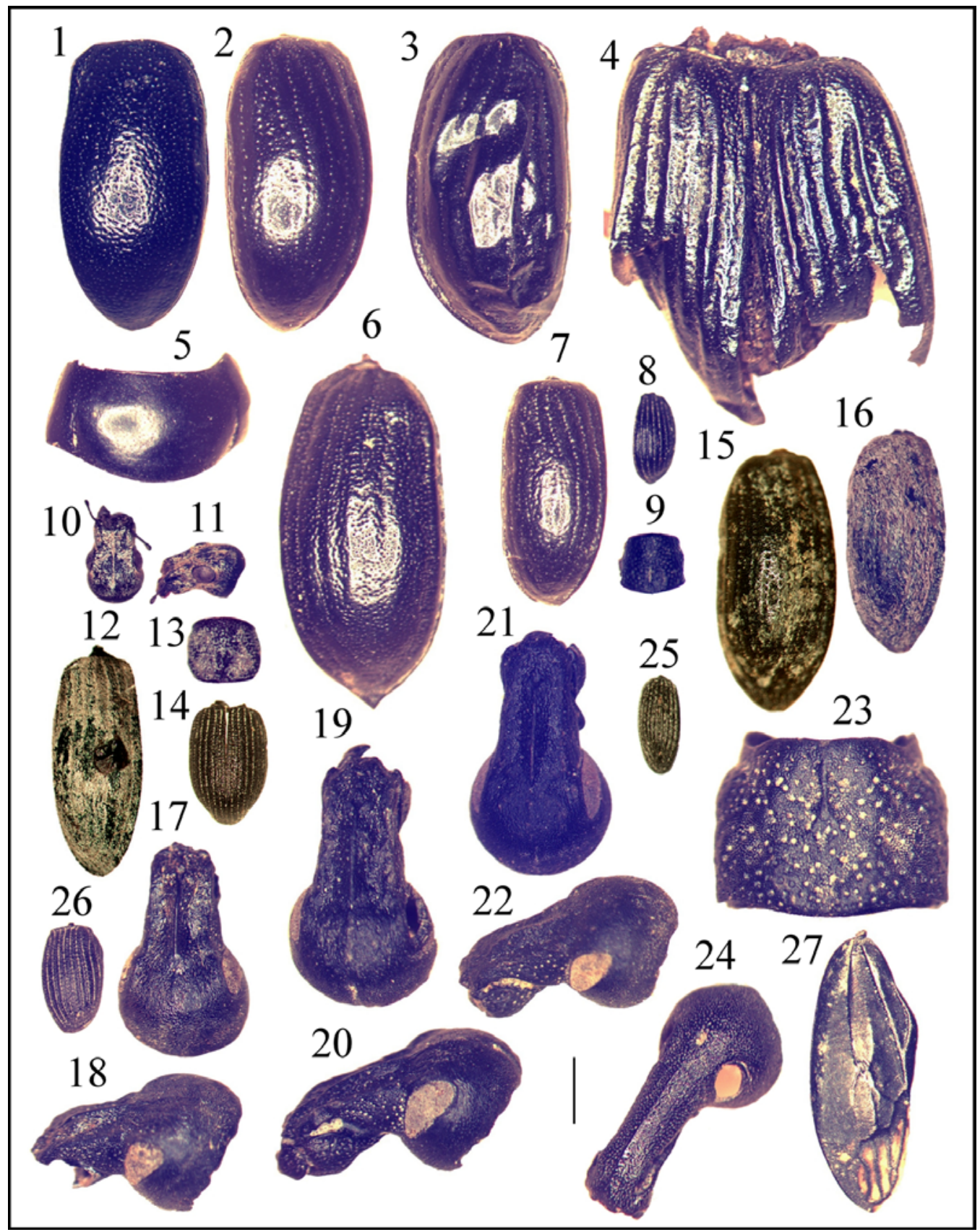

Fig. 8. Fossil insects (Chrysomelidae - Saldidae) from the Titaluk river site.

1 - Chrysolina basilaris, 2 - C. septentrionalis, 3 - C. magniceps, 4 - C. subsulcata, 5 - Chrysolina sp., 6 Chrysomela blaisdelli, 7 - Phratora kenaiensis, 8 - Mesotrichapion alaskanum, 9 - M. cyanitinctum, 10, 11, 12 Sitona aquilonius, 13, 14 - Lepidophorus thulius, 15 - Hypera castor, $16-H$. diversipunctata, 17, 18 Stephanocleonus confusus, 19, 20 - Coniocleonus parshus, 21, 22 - C. zherichini, 23 - Coniocleonus sp., $24-$ Lepyrus nordenskioeldi, 25 - Isochnus arcticus, 26 - Ceutorhynchus subpubescens, 27 - Chiloxanthus sp.; 1 T03-B06; 2, 3, 7, 15, 16, 17, 18, 24, - T03-B13; 4 - T03-B15; 8, 9 - T03-B13a; 10, 11, 13, 19, 20, 21, 22, 23 T03-B18; 12, 14, 26 - T03-B07a; 25 -T03-B03; 27 - T03-B12; 1, 3, 6, 8 - right elytron; 2, 7, 12, 15, 16, 25, 27 left elytron; 4, 14 - both elytra; 4, 9, 13, 23 - pronotum; 10, 11, 17, 18, 19, 20, 21, 22, 24 - head. Scale bar $1 \mathrm{~mm}$. 
cus, make the fossil Pleistocene assemblages quite peculiar. There is certainly no modern analogue for this combination of species.

Single specimens of forest beetles were found in the lower part of the sandy unit. The sample T-B15 contains the single remains of the weevil Hylobius pinicola and the bark beetle Scierus annectans, and T-B03 also contained H. pinicola. These species are indicators of coniferous forest. Despite the possibility of reworking, we are forced to conclude that a warming episode took place in the region sometime during the Pleistocene.

The Yedoma-analogue unit (C), in contrast with lower gravel and sand, seemed devoid of insect remains, but by screening a couple of hundred $\mathrm{kg}$ of sediment we obtained two good assemblages from three samples (Figs 5-6, Table 2). The proportion of the steppe-tundra group in sample T-B08a is highest in the section - 24\%. Otherwise, the species composition and general ecological structure of these two assemblages was similar to the previous ones.

The Poplar Beds unit (D) was found in two profiles at Titaluk River (Figs 4-6, Table 2). In spite of the climatic amelioration that apparently allowed poplar to invade the North Slope, the general species and ecological composition of the insect assemblages remained the same. A similar picture has been observed in the insect fossil assemblages from the Populus Beds of the Ikpikpuk River (Nelson, Carter, 1987). Xerophilous insects dominated most of the assemblages, and the steppe-tundra still group played significant role. The Arctic group became less evident but single remains of Isochnus arcticus were still present in each sample. The main difference was observed in samples T-B10, 11, and 12 from the first profile (Fig. 4). This section consisted of bluish-gray fine sand with layers of well-preserved poplar leaves. We noted a sharp increase in the water-riparian group, especially in sample T-B11 (Fig. 6, Table 2).
They are represented mostly by the diving beetles of the genera Agabus and Hydroporus.

Sample T-B12 is only one in the section that contained fossils of the winter eggs (ephippia) of water fleas (Fig. 9). We recognized numerous ephippia of Daphnia cf. longispina O.F. Müller and Simocephalus sp. (Fam. Daphniidae Straus). Exact species identification of ephippia is difficult (Kotov, 2015). Different taxa of the $D$. longispina can live in a variety of fresh water habitats (Benzie, 2005). Members of the genus Simocephalus are typical inhabitants of shallow, vegetated margins of standing water (Orlova-Bienkowskaja, 2001). The fossils water fleas indicate the presence of temporary floodplain pools oxbows, but not the river itself.

The second profile (Fig. 5) probably represents a river channel. It overlay the older unit with a clear unconformity. Dynamic fluvial conditions supported reworking and mixing of older and younger fossils.

\section{Discussion}

Nelson $(1982,1986)$ started the investigation of North Slope fossil insects and vegetation in the 1980s. He proposed the following scenario of environmental changes:

1 - before $40 \mathrm{ka}$ : Environment similar to modern with less abundant shrub vegetation and more open surfaces. Climate was cooler and drier than today.

2 - between 40 and $25 \mathrm{ka}$ : decreasing moisture and temperature.

3 - Early Holocene. Populus balsamifera and a few other species were present north of their modern limits. Climate was warmer than today but the landscape was discontinuously vegetated and summer precipitation may have been infrequent.

Our climate estimation using MCR method is shown in the Table 3. In general, the recon-

Рис. 8. Ископаемые насекомые (Chrysomelidae - Saldidae) из разреза на реке Титалук.

1 - T03-B06; 2, 3, 7, 15, 16, 17, 18, 24, - T03-B13; 4- T03-B15; 8, 9- T03-B13a; 10, 11, 13, 19, 20, 21, 22, 23 - Т03-В18; 12, 14, 26 - Т03-В07a; 25-Т03-В03; 27 - Т03-В12; 1, 3, 6, 8 - правое надкрылье; 2, 7, 12, $15,16,25,27$ - левое надкрылье; 4, 14 - соединенные надкрылья; 4, 9, 13, 23 - переднеспинка; 10, 11, 17, 18, 19, 20, 21, 22, 24 - голова. Шкала 1 мм. 


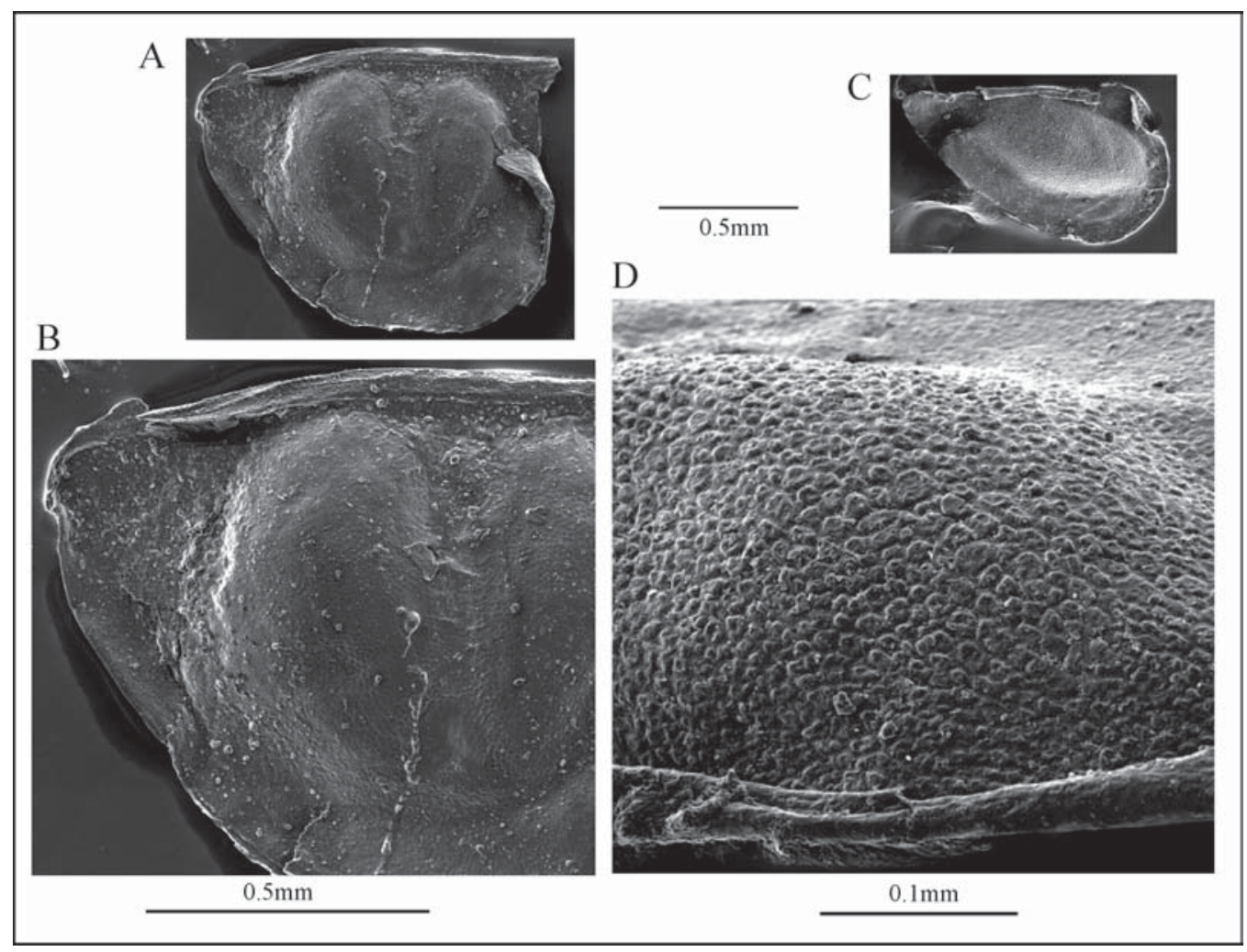

Fig. 9. SEM photos of daphniid ephippia (Cladocera) from sample T03-B-12.

A-B - Daphnia cf. longispina C-D - Simocephalus sp.; A, C - general view, B, D - surface sculpture.

Fig. 9. Фотографии со сканирующего электронного микроскопа эфиппиумов дафнид (Cladocera) из образца Т03-В-12.

A-B - Daphnia cf. longispina; А, С - общий вид, В, D - скульптура поверхности.

struction shows lower than recent temperatures during all time intervals including the Early Holocene. The warming signal in the sample TB-09 is not much reliable because the examined insect assemblage is small. Another assemblage what provides warmer than recent temperature T-B-14 from the lower unit A is big but thermophilous species which affected the MCR result is only one - Cymindis unicolor. This species is more likely absent in the studied area recently, R Nelson (Nelson, 2001) who worked exactly at the Titaluk River as well as in other sites of the North Slope did not collected it; we also did not find the species in the modern environment. $C$. unicolor lives in the tundra and alpine treeless environment on well-drained soil. It occurs from Newfoundland to southwest Yukon and in moun- tains across Canada and western USA (Bousquet, 2012).

The rest of species composition is similar to these of other samples. From the other hand we found several forest species in the lower unit. These single evidences indicate probably eroded interglacial layer.

Climate estimation of the Poplar Beds using fossil insects shows lower temperature (7.5 to $10^{\circ} \mathrm{C}$ than mean July temperatures 14 to $15^{\circ} \mathrm{C}$ reconstructed using Populus balsamifera (Nelson, Carter, 1987). Thermophilous insects did not follow the poplar groves, and it is hard to explain. Maybe the Brook Ridge prevents the spreading of southern fauna to this area while poplar seeds which are adapted to wind transportation were able to overcome this barrier. 
Table 3. Reconstruction using MCR of mean July (TMAX), mean January (TMIN), and differences with modern temperature of the nearest weather station - Umiat, $\operatorname{AK}\left(69.22^{\circ} \mathrm{N}, 152.08^{\circ} \mathrm{W}\right)$.

Таблица 3. Реконструкция методом MCR среднеиюльских температур (TMAX) и среднеянварских (TMIN) и разница с современной температурой по данным ближайшей метеостанции Умиат, Аляска $\left(69.22^{\circ} \mathrm{N}, 152.08^{\circ} \mathrm{W}\right)$.

\begin{tabular}{llllll}
\hline recent & 10 & -30 & No. spp & & \\
Sample & TMAX & TMIN & used & $\Delta$ TMAX & $\Delta$ TMIN \\
\hline Ikpikpuk B-0-4 Unit A & $7.5-9.5$ & -33.5 to -28.5 & 15 & -2.5 to -0.5 & -3.5 to +1.5 \\
Ikpikpuk B-0-4a Unit A & $8.5-9$ & -31 to -29.5 & 20 & -1.5 to -1 & -1 to +0.5 \\
Ikpikpuk B-0-5 Unit A & $8-9.5$ & -33 to -28.5 & 14 & -2 to -0.5 & -3 to +0.5 \\
Titaluk B-0-1 Unit A & $5.5-8$ & -31.5 to -26 & 16 & -4.5 to -2 & -1.5 to +4 \\
Titaluk B-0-2 Unit A & $7-9$ & -31.5 to -24 & 10 & -3 to -1 & -1.5 to +6 \\
Titaluk B-0-13 Unit A & $9-10$ & -31.5 to -28.5 & 22 & -1 to 0 & -1.5 to +1.5 \\
Titaluk B-0-13a Unit A & $8.5-9$ & -31 to -29 & 21 & -1.5 to -1 & -1 to +1 \\
Titaluk B-0-14 Unit A & $11-11.5$ & -21.5 to -20.5 & 15 & +1 to +1.5 & +8.5 to +9.5 \\
Titaluk B-0-3 Unit B & $8.5-9$ & -31 to -29.5 & 17 & -1.5 to -1 & -1 to +0.5 \\
Titaluk B-0-4 Unit B & $8-10$ & -31 to -22 & 9 & -2 to 0 & -1 to +8 \\
Titaluk B-0-6 Unit B & $8.5-9$ & -31 to -29.5 & 15 & -1.5 to -1 & -1 to +0.5 \\
Titaluk B-0-7 Unit B & $8.5-9$ & -31 to -29.5 & 12 & -1.5 to -1 & -1 to +0.5 \\
Titaluk B-0-7a Unit B & $8.5-9$ & -31.5 to -29 & 24 & -1.5 to -1 & -1.5 to +1 \\
Titaluk B-0-15 Unit B & $8-9$ & -31 to -29 & 28 & -2 to -1 & -1 to +1 \\
Titaluk B-0-8a Unit C & $8-8.5$ & -32 to -30.5 & 18 & -2 to -1.5 & -2 to -0.5 \\
Titaluk B-0-8b Unit C & $8.5-9$ & -31 to -29.5 & 18 & -1.5 to -1 & -1 to -0.5 \\
Titaluk B-0-9 Unit C & $9.5-10$ & -25 to -22 & 6 & -0.5 to 0 & +5 to +8 \\
Titaluk B-0-11 Unit D & $7.5-9$ & -32 to -29 & 9 & -2.5 to -1 & -2 to +1 \\
Titaluk B-0-11a Unit D & $8.5-9$ & -31 to -29 & 8 & -1.5 to -1 & -1 to +1 \\
Titaluk B-0-12 Unit D & $8.5-9$ & -31 to 29.5 & 13 & -1.5 to -1 & -1 to +0.5 \\
Titaluk B-0-16 Unit D & $8-10$ & -33 to -25 & 10 & -2 to 0 & -3 to +5 \\
Titaluk B-0-17 Unit D & $8.5-9.5$ & -31 to -26.5 & 17 & -1.5 to -0.5 & -1 to +3.5 \\
Titaluk B-0-18 Unit D & $9-9.5$ & -30 to -29 & 11 & -1 to -0.5 & 0 to +1 \\
\hline & & & & & \\
\hline
\end{tabular}

Our results support Nelson's conclusions about drier-than-modern climate and warming in the early Holocene. He noticed that the main surface (away from the river valley) was probably unstable, dry, sandy, and covered by discontinuous vegetation. Such surface conditions also existed during cold Pleistocene intervals, as well as in the early Holocene (Nelson, Carter, 1987).

The difference between our reconstructions and those of Nelson is that he never used the term "steppe-tundra". This concept became much more widely discussed in the 1990s, well after Nelson's dissertation was finished. Perhaps he was also influenced by the plant macrofossil and pollen results, as plant remains rarely indicate the steppe-tundra environment. Also, his list of identified insects only included tundra species, and Coniocleonus specimens were rare in his assemblages, and were simply identified as Cleonus sp. The taxonomic understanding of this group of weevils has advanced considerably in the last 40 years. Our specific identification of Stephanocleonus confusus and Conio- 
cleonus parshus shows that some species were present north of their modern limits, even during the Pleistocene. This situation is more typical of Western Beringian steppe-tundra entomofaunas (Kiselev, 1981; Kuzmina, 2015), of which Nelson had little or no knowledge during the 1980s, when nearly all Russian research in this field was published in the Russian language, and was virtually unobtainable in the US.

The abundance of xerophilous species, especially Morychus and Lepidophorus lineaticollis has been recorded in the Ikpikpuk (Nelson, Carter, 1987) and Titaluk Populus Beds. The Titaluk faunal succession suggests that the insect fauna changed little at the PleistoceneHolocene transition. Steppe-tundra environment survived the early Holocene warming in this region, perhaps because of the cold climate and lack of well developed forest in this northern locality. A similar situation was observed in northern localities in Western Beringia (Andreev et al., 2009; Kuzmina, 2015) while in southern regions, such as Klondike Gold Field, the Pleistocene-Holocene transition is sharp and the steppe-tundra fauna disappeared from the record almost completely (Kuzmina, unpublished).

Single forest insects, wood and stumps were found occasionally in the Pleistocene part of the section. We can not correlate them with specific layers, and perhaps most of them were reworked from older deposits of unknown age. It is probable that forest-associated insect remains were eroded out of sediments from a Pleistocene warm interval, such as the Last Interglacial. It is hard to verify easily because the radiocarbon dates of the wood from the lower units (Table 1) are infinitive or close to the method limit.

Open, sandy surfaces helped to maintain high soil temperatures that are critical for the development of many thermophilous insects. For instance Cleonini weevils are very sensitive to soil temperature (Berman et al., 2011). They need significant amounts of warmth for the development of larvae in the soil. Arctic regions could provide the necessary sum of positive soil temperatures during the summer months when daylight hours are long or continuous. The ab- sence of trees and moss cover is also critical for the maintenance of soil warming. Under a dry Pleistocene - Early Holocene climate regime, thermophilous Cleonini weevils flourished in the northern Beringian steppe-tundra.

Continuous eolian sand deposition played an important role in the local environment of the study sites. Late Pleistocene eolian activity affected the region widely. As discussed above, a large sand dune area (sand sea) is located very exactly near to the studied sections (Carter, 1981). Another sand deposit of eolian origin, the Kittigazuit Formation, is known from the Tuktoyaktuk Coastlands in the northern part of the Canadian Northwest Territories (Murton, 1996). Fossil insect assemblages from Kittigazuit Formation (Dallimore et al., 1987, Murton et al., 2017) are similar in species composition to those found in the Ikpikpuk-Titaluk area.

Wind itself helped to maintain favourable environmental conditions for key Pleistocene insect species. Observations of Berman (1990) in northeastern Siberia allow us to reconstruct the environmental requirements of the key Pleistocene species Morychus viridis Kuzm. et Korot., 1987. This pill beetle feeds on certain minute mosses that can develop only on dry open surfaces. Wind helps to develop such surfaces by preventing the development of the dense moss-lichen cover found in the tundra and in the boreal forest. Eastern Beringian $M$. aff. aeneolus probably occupied similar habitats to M. viridis (Kuzmina, unpublished).

The long-term presence of such Arctic insects as Isochnus arcticus and several species of Chrysolina may seem to contradict the presence of other faunal elements. This seeming contradiction may be explained in two different ways: (1) Modern species with high Arctic distribution are relicts of the Pleistocene steppe-tundra community. They, being cold-resistant, survive now in northern open treeless landscapes where they avoid competition with southern species. Indeed, almost all Pleistocene steppe-tundra assemblages contain Arctic species (Kuzmina, Matthews, 2012). They were equitable members of this non-analogue community. (2) Arctic species were indifferent to climate but sensitive 
for discontinuous vegetation cover. This suggestion needs specification - the Arctic group is present in most of the fossil assemblages, but it only became dominant during the coldest Pleistocene intervals (Sher et al., 2005) and even then this dominance occurred mostly in northernmost sites. Under this scenario, the arctic species indicate substantial cooling if their numbers increase dramatically.

The task of "looking at the Eastern Beringian section through Western Beringian eyes" was successfully realized. We made certain that the Siberian pill beetle, Morychus viridis, and the North American fossil specimens of Morychus are indeed different species. Furthermore, we were able to verify that the North American fossil Cleonini specimens belong to a regional species, and that the diversity of Pterostichus (Cryobius) species in Eastern Beringian fossil insect faunas is greater than the diversity of this group in Siberian faunas.

North Slope fossil insect assemblages show some differences between Western and Eastern Beringian steppe-tundra communities. Steppe elements are more prominent in Siberia; the Alaskan North Slope steppe-tundra was more "tundra-like". The dominant beetle taxon of the local community is the weevil Lepidophorus lineaticollis; Morychus plays significant but secondary role. In Siberia L. lineaticollis is absent, and Morychus viridis specimens often comprise $80-90 \%$ of assemblage.

Many species recorded in the North Slope fossil assemblages have Amphi-beringian distributions (i.e., they are found today on both sides of the Bering Strait). These include Bembidion umiatense, Dicheirotrichus mannerheimi, Harpalus vittatus alaskensis, Poecilus nearcticus, Pterostichus pinguedineus, $P$. brevicornis, $P$. ventricosus, $P$. agonus, $P$. costatus, P. vermiculosus, $P$. sublaevis, Stereocerus haematopus, Amara alpina, Agabus moestus, Helophorus splendidus, Micralymma brevilingue, Tachinus brevipennis, Chrysolina septentrionalis, Ch. subsulcata, Hypera diversipunctata, Coniocleonus zherichini, Lepyrus nordenskioeldi, and Isochnus arcticus. Lepidophorus thulius was Amphi-Beringian in the Pleistocene but today it is found live only in North America.

Our study of Eastern Beringia fossil insects has allowed us to recognize another important feature. Many cold-phase Pleistocene insect assemblages from the Yukon (Kuzmina, Matthews, 2012; Matthews et al., 2019) are dominated by the xerophilous weevil Connatichela artemisiae And., 1984. This species has not been found in any of the North Slope samples; it is also absent in fossil assemblages from Western Beringia. This weevil feeds on Artemisia. It appears that Artemisia-dominated steppes developed only in the interior regions of Eastern Beringia during the Last glaciation.

The vegetation cover of the North Slope steppe-tundra probably included various grasses, as indicated by the presence of Amara alpi$n a$. The presence of herbs including legumes is indicated by the presence of Coniocleonus, Sitona, and Hypera diversipunctata. Buttercups are the host plant of Chrysolina septentrionalis, and cruciferous plants are eaten by Chrysolina tolli and Ceutorhynchus subpubescens. Tall shrub willows are attacked by Lepyrus canadensis, L. gemellus, L. nordenskioeldi, and Chrysomela blaisdelli, while dwarf willow is the host plant for Isochnus arcticus. The bark beetles Hylobius pinicola, Scierus annectans, and Scolytus piceae feed on coniferous trees.

\section{Conclusions}

This study is contributed to our knowledge of the Pleistocene-Early Holocene insect faunas of the Alaskan North Slope, and the fossil evidence provided the basis for our paleoenvironmental reconstructions, as well as yielding new information concerning the nature of the nowextinct steppe-tundra community. The climate during the Late Pleistocene and Early Holocene allowed the steppe-tundra insect fauna to survive in situ, in spite of global warming at the end of the Last Glaciation. Early Holocene warming and the advance of poplar forest onto the North Slope did not greatly affect the regional insect fauna, perhaps because poplar trees were sparse on the landscape, and did not shade all the surfaces. Low moisture was probably more im- 
portant in maintaining regional steppe-tundra temperature regime.

Herbs and grasses dominated the local vegetation cover and Artemisia did not play a significant role here. Tall willow shrubs occupied riparian habitats, while dwarf willow probably grew on upland surfaces. Wind-driven sand deposition probably helped maintain the steppetundra community on the North Slope.

The species and ecological requirements of the North Slope fossil beetle assemblages indicate a specific local environment that differed from more southerly localities in Beringia, thus suggesting regional zonation of Beringian ecosystems.

The steppe-tundra insect fauna of the North Slope is both similar to and different from those described from Northeastern Siberia. The two faunas share may species in common but the xerophilous elements are mostly different. These faunal differences were most likely due to differences in the climates of the two regions. It appears that northern Western Beringian climates were drier than those of northern Eastern Beringia.

\section{Acknowledgments}

The field research was much assisted by the National Parks Service of the USA. We thank the Russian Foundation for Basic Research (grants 04-04-48770 for SK), Leverhulme Foundation, F/07 537T (grant for SE) and Russian Government Program of Competitive Growth of Kazan Federal University for support of palaeoentomological studies. This study of Beringian cladocerans is supported by the Russian Foundation for Basic Research (grant 18-0400398). We appreciate kindness of Pamela Groves and Daniel Mann who recognized our field location. Special thank to staff of the Canadian national collection of insects, arachnids and nematodes (CNC) for their hospitality and patience.

\section{References}

Ager T. 1982. Vegetational history of Western Alaska during the Wisconsin Glacial Interval and the Ho- locene // D.M. Hopkins, J.V. Matthews Jr., C.E.Schweger, S.B. Young (eds.). Paleoecology of Beringia. New York: Academic Press. P.75-94.

Alfimov A.V., Berman D.I. 2001. Beringian climate during the Late Pleistocene and Holocene // Quaternary Sci. Rev. Vol.1. No.20. P.127-134.

Alfimov A.V., Berman D.I., Sher A.V. 2003. [Tundrasteppe assemblages of insects and the climatic reconstruction for the lower Kolyma in the Late Pleistocene] // Zool. Zhurn. Vol.82. No.2. P.281-300 [in Russian].

Alonso-Zarazaga M.A. 1999. A World catalogue of families and genera of Curculionoidea (Insecta: Coleoptera) (excepting Scolytidae and Platypodidae). Barcelona: Entomopraxis. 315 p.

Andreev A.A., Grosse G., Schirrmeister L., Kuznetsova T.V., Kuzmina S.A., Bobrov A.A., Tarasov P.E., Novenko E.Y., Meyer H., Derevyagin A.Y., Kienast F., Bryantseva A., Kunitsky V.V. 2009. Weichselian and Holocene palaeoenvironmental history of the Bol'shoy Lyakhovsky Island, New Siberian Archipelago, Arctic Siberia // Boreas. Vol.38. No.1. P.72-110.

Anderson P.M. 1985. Late Quaternary vegetational change in the Kotzebue Sound area, Northwest Alaska // Quaternary Res. Vol.24. P.307-321.

Anderson P.M., Brubaker L.B. 1994. Vegetation history of north-central Alaska: A mapped summary of LateQuaternary pollen data// Quaternary Sci. Rev. Vol.13. P.71-92.

Anderson P.M., Bartlein P.J., Brubaker L.B., Gajewski K., Ritchie J.C. 1989. Modern analogues of Late Quaternary pollen spectra from the Western Interior of North America // J. Biogeogr. Vol.16. P.573-596.

Anderson R.S. 1987. Systematics, phylogeny and biogeography of New World weevils traditionally of the tribe Cleonini (Coleoptera: Curculionidae; Cleoninae) // Quaest. Entomol. No.23. P.431-709.

Anderson R.S. 1989. New synonymy in North American Stephanocleonus Motschulsky (Coleoptera: Curculionidae) // Coleopterists Bull. Vol.43. No.1. P.93.

Benzie J.A.H. 2005. The genus Daphnia (including Daphniopsis) (Anomopoda: Daphniidae) // Guides to the identification of the microinvertebrates of the continental waters of the world. Vol.21. Leiden: Kenobi Productions and Ghent: Backhuys Publishers. 376 p.

Berman D.I. 1990. [Modern habitats of a pill beetle Morychus viridis (Coleoptera, Byrrhidae) and the reconstruction of the Pleistocene environment in the NorthEast of the USSR] // Doklady AN SSSR. Vol.310. No.4. P.1021-1023 [in Russian].

Berman D.I., Alfimov A.V., Korotyaev B.A. 2002. [Xerophilous arthoropods in the tundra-steppe of the site Utesiki (Chukotka)] // Zool. Zhurn. Vol.81. No.4. P.444-450 [in Russian].

Berman D., Alfimov A., Kuzmina S. 2011. Invertebrates of the relict steppe ecosystems of Beringia, and the reconstruction of Pleistocene landscapes // Quaternary Sci. Rev. Vol.30. No.17-18. P.2200-2219.

Briner J.P., Kaufman D.S. 2008. Late Pleistocene mountain glaciation in Alaska: key chronologies // J. Quaternary Sci. Vol.23. P.659-670. 
Bockheim J.G., Hinkel K.M. 2005. Characteristics and signi? cance of the transition zone in drained thawlake basins of the Arctic Coastal Plain, Alaska // Arctic. Vol.58. No.40. P.6-417.

Bockheim J.G., O’Brien J., Munroe J., Hinkel K. 2003. Factors affecting the distribution of Populus balsamifera on the North Slope of Alaska, U.S.A. // Arct. Antarct. Alp. Res. Vol.35. P.331-340.

Bousquet Y. 2012. Catalogue of Geadephaga (Coleoptera, Adephaga) of America, north of Mexico // ZooKeys. Vol.245. P.1-1722.

Carter L.D. 1981. A Pleistocene sand sea on the Alaskan arctic coastal plain // Science. Vol.211. P.381-383.

Carter L.D. 1993. Late-Pleistocene eolian sand sheets in Alaska // Quaternary Res. Vol.34. P.269-281.

Colinvaux P.A. 1967. Quaternary vegetational history of Arctic Alaska // D.M. Hopkins (ed.). The Bering Land Bridge. Stanford: Stanford University Press. P.207231.

Dallimore S.R., Wolfe S.A., Matthews Jr., J.V., Vincent J.S. 1997. Mid Wisconsinan eolian deposits of the Kittigazuit Formation, Tuktoyaktuk Coastlands, Northwest Territories, Canada // Can. J. Earth Sci. Vol.34. P.1421-1441.

Edwards M.E., Barker Jr., E.D. 1994. Climate and vegetation in northeastern Alaska, 18,000 yr B.P. - present // Palaeogeogr. Palaeoclimatol. Palaeoecol. Vol.109. P.127-135.

Elias S.A. 2001. Mutual climatic range reconstructions of seasonal temperatures based on Late Pleistocene fossil beetle assemblages in eastern Beringia // Quaternary Sci. Rev. Vol.20. P.77-91.

Finkenbinder M.S., Abbott M.B., Finney B.P., Stoner J.S., Dorfman J.M. 2015. A multi-proxy reconstruction of environmental change spanning the last 37,000 years from Burial Lake, Arctic Alaska // Quaternary Sci. Rev. Vol.126. P.227-241.

Gaglioti B.V, Mann D.H., Wooller M.J., Jones B.M., Wiles G.C., Groves P., Kunz M.L., Baughman C.A., Reanier R.E. 2017. Younger-Dryas cooling and seaice feedbacks were prominent features of the Pleistocene-Holocene transition in Arctic Alaska // Quaternary Sci. Rev. Vol.169. P.330-343.

Gaglioti B.V, Mann D.H., Groves P., Kunz M.L., Farquharson L.M., Reanier R.E., Jones B.M., Wooller M.J. 2018. Aeolian stratigraphy describes ice-age paleoenvironments in unglaciated Arctic Alaska // Quaternary Sci. Rev. Vol.182. P.175-190.

Goetcheus V.G. 2001. A window to the past: macrofossil remains from an 18,000 year old buried surface, Seward Peninsula, Alaska. M.Sci. Thesis. Fairbanks: University of Alaska. 176 p.

Goetcheus V.G., Birks H.H. 2001. Full-glacial upland tundra vegetation preserved under tephra in the Beringia National Park, Seward Peninsula, Alaska // Quaternary Sci. Rev. Vol.20. P.135-147.

Goetcheus V.G., Hopkins D.M., Edwards M.E., Mann D.H. 1994. Window on the Bering Land Bridge: a 17,000 yr BP paleosurface on the Seward Peninsula, Alaska // Curr. Res. Pleistocene. Vol.11. P.131-132.
Grosse G., Schirrmeister L., Siegert C., Kunitsky V.V., Slagoda E.A., Andreev A.A., Dereviagyn A.Y. 2007. Geological and geomorphological evolution of a sedimentary periglacial landscape in Northeast Siberia during the Late Quaternary // Geomorphology. Vol.86. P.25-51.

Guthrie R.D. 1990. Frozen Fauna of the Mammoth Steppe: The Story of Blue Babe. Chicago: University of Chicago Press. 323 p.

Guthrie R.D. 2001. Origin and causes of the mammoth steppe: a story of cloud cover, woolly mammal tooth pits, buckles, and inside-out Beringia // Quaternary Sci. Rev. Vol.20. P.549-574.

Guthrie R.D., Stoker S. 1990. Paleoecological Significance of Mummified Remains of Pleistocene Horses from the North Slope of the Brooks Range, Alaska // Arctic. Vol.43. No.3. P.267-27.

Hopkins D.M. 1972. The paleogeography and climatic history of Beringia during late Cenozoic time // Internord. Vol.12. P.121-15.

Hultén E. 1937. Outline of the History of Arctic and Boreal Biota During the Quaternary Period. Thule, Stockholm: Bokförlags Aktie-Bolaget. 168 p.

Johnson P.J. 1991. Taxonomic notes, new records, and a key to the adults of North American Byrrhidae (Coleoptera) // Proc. Entomol. Soc. Wash. Vol.93. No.2. P.322-332.

Kane D.L., Youcha E.K., Stuefer S.L., Myerchin-Tape G., Lamb E., Homan J.W., Gieck R.E., Schnabel W.E., Toniolo H. 2014. Hydrology and Meteorology of the Central Alaskan Arctic: Data Collection and Analysis, Report INE/WERC 14.05. Fairbanks, Alaska: University of Alaska Fairbanks, Water and Environmental Research Center. 168 p.

Kanevskiy M., Shur Y., Fortier D., Jorgenson M.T., Stephani E. 2011. Cryostratigraphy of late Pleistocene syngenetic permafrost (Yedoma) in northern Alaska, Itkillik River exposure // Quaternary Res. Vol.75. P.584-596.

Kanevskiy M., Shur Yu., Strauss J., Jorgenson T., Fortier D., Stephani E., Vasiliev A. 2016. Patterns and rates of riverbank erosion involving ice-rich permafrost (Yedoma) in northern Alaska // Geomorphology. Vol.253. P.370-384.

Kaplina T.N., Lozhkin A.V. 1982. [History of vegetation development in the Coastal Lowlands of Yakutia during the Holocene] // A.A. Velichko (ed.). Razvitie prirody territorii SSSR v pozdnem pleistotsene i golotsene. Moscow: Nauka. P.207-220 [in Russian].

Kaufman D.S., Manley W.F. 2004. Pleistocene Maximum and Late Wisconsin glacier extents across Alaska, USA // J. Ehlers, P.L. Gibbard (eds.). Quaternary Glaciations: Extent and Chronology. Part II: North America. Amsterdam: Elsevier. P.9-27.

Kaufman D.S., Ager T.A., Anderson N.J., Anderson P.M., Andrews J.T., Bartlein P.J., Brubaker L.B., Coats L.L., Cwynar L.C., Duvall M.L., Dyke A.S., Edwards M.E., Eisner W.R., Gajewski K., Geirsdottir A., Hu F.S., Jennings A.E., Kaplan M.R., Kerwin M.W., Lozhkin A.V., MacDonald G.M., Miller G.H., Mock 
C.J., Oswald W.W., Otto-Bliesner B.L., Porinchu D.F., Ruhland K., Smol J.P., Steig E.J., Wolfe B.B. 2004. Holocene thermal maximum in the western Arctic (0-180 W) // Quaternary Sci. Rev. Vol.23. P.529-560.

Kienast F. 2006. Plant macrofossil records. Arctic Eurasia // S. Elias (ed.). Encyclopedia of Quaternary Science. Amsterdam: Elsevier. P.2422-2434.

Kiselev S.V. 1981. [Late Cenozoic Coleoptera of NorthEast Siberia]. Moscow: Nauka. 116 p. [In Russian]

Kotov A.A. 2015. A critical review of the current taxonomy of the genus Daphnia O. F. Müller, $1785 / /$ Zootaxa. Vol.3911. No.2. P.184-200.

Kuzmina S.A. 2015. Quaternary Insects and Environment of the Northeastern Asia // Paleontol. J. Supplement. Vol.49. No.7. P.1-189.

Kuzmina S.A., Elias S.A. 2019. Andrei V. Sher and his role in Quaternary invertebrate study // Invertebrate Zool. Vol.16. No.2. P.79-88.

Kuzmina S., Sher A. 2006. Some features of the Holocene insect faunas of northeastern Siberia // Quaternary Sci. Rev. Vol.25. P.1790-1820.

Kuzmina S., Elias S., Matheus P., Storer J.E., Sher A. 2008. Paleoenvironmental reconstruction of the Last Glacial Maximum, inferred from insect fossils from a buried soil at Tempest Lake, Seward Peninsula, Alaska//Palaeogeogr. Palaeoclimatol. Palaeoecol. Vol.267. P.245-255.

Kuzmina S., Froese D.G., Jensen B.J.L., Hall E., Zazula G.D. 2014. Middle Pleistocene (MIS 7) to Holocene fossil insect assemblages from the Old Crow basin, northern Yukon, Canada// Quaternary Intern. Vol.341. P.216-242.

Kuzmina S., Matthews J.V. 2012. [Late Cenozoic insects of Beringia] // Euroasian Entomol. J. Vol.11. Suppl.1. P.9-97 [in Russian].

Lapointe L.E., Talbot J., Fortier D., Fréchette B., Strauss J., Kanevskiy M., Shur Yu. 2017. Middle to late Wisconsinan climate and ecological changes in northern Alaska: Evidences from the Itkillik River Yedoma // Palaeogeogr. Palaeoclimatol. Palaeoecol. Vol.485. P.906-916.

Lindroth C.H. 1966. The ground-beetles (Carabidae, excl. Cicindelinae) of Canada and Alaska. Part 4 // Opuscula Entomol. Suppl. Vol.29. P.409-648.

MacDonald G.M., Velichko A.A., Kremenetski C.V., Borisova C.K., Goleva A.A., Andreev A.A., Cwynar L.C., Riding R.T., Forman S.L., Edvards T.W.D., Aravena R., Hammarlund D., Szeicz J., Gattaulin V.N. 2000. Holocene treeline history and climate change across northern Eurasia // Quarternary Res. Vol.53. P.302-311.

Mann D.H., Peteet D.M., Reanier R.E., Kunz M.L. 2002. Responses of an arctic landscape to Lateglacial and early Holocene climatic changes: the importance of moisture// Quaternary Sci. Rev. Vol.21. P.997-1021.

Mann D.H., Groves P., Reanier R.E., Kunz M.L. 2010. Floodplains, permafrost, cottonwood trees, and peat: What happened the last time climate warmed suddenly in arctic Alaska? // Quaternary Sci. Rev. Vol.29. P.3812-3830.
Mann D.H., Groves P., Kunz M.L., Reanier R.E., Gaglioti B.V. 2013. Ice-age megafauna in Arctic Alaska: extinction, invasion, survival // Quaternary Sci. Rev. Vol.70. P.91-108.

Mann D.H., Groves P. Reanier R.E., Gaglioti B.V., Kunz M.L., Shapiro B. 2015. Life and extinction of megafauna in the ice-age Arctic // PNAS. Vol.112. No.46. P.14301-14306.

Matthews J.V. 1968. A paleoenvironmental analysis of three Late Pleistocene coleopterous assemblages from Fairbanks, Alaska // Quaest. Entomol. Vol.4. P.202224.

Matthews J.V. 1979. Beringia during the Late Pleistocene: arctic-steppe or discontinuous herb-tundra? A review of the paleontological evidence // Paleoecology of the arctic-steppe mammoth biome; 8-17 June 1979. Preprint, No.81. Ottawa: Geological Survey of Canada. $60 \mathrm{p}$.

Matthews J.V. 1983. A Method for Comparison of Northern Fossil Insect Assemblages // Géogr. Phys. Quatern. Vol.37. No.3. P.297-306.

Matthews J.V. Jr., Telka A., Kuzmina S.A. 2019. Late Neogene insect and other invertebrate fossils from Alaska and Arctic/Subarctic Canada // Invertebrate Zool. Vol.16. No.2. P.126-153.

Matthews J.V. Jr., Telka A. 1997. Insect Fossils from the Yukon // H.V. Danks, J.A. Downes (eds.): Insects of the Yukon (Terrestrial Arthropods). Ottawa: Biological Survey of Canada. P.911-962.

Meregalli M. 2017. World catalogue of the Curculionidae: Lixinae: Cleonini. Available from: http://weevil.info/ content/world-catalogue-curculionidae-lixinae-cleonini\# (accessed 19 July 2017).

Meregalli M., Fremuth J. 2013. tribe Cleonini // I. Löbl, A. Smetana (eds.). Catalogue of Palaearctic Coleoptera. Vol.8. Curculionoidea II. Leiden: Brill. P.437-456.

Murton J.B. 1996. Morphology and paleoenvironmental significance of Quaternary sand veins, sand wedges, and composite wedges, Tuktoyaktuk Coastlands, western Arctic Canada // J. Sediment. Res. Vol.66. P.1725.

Murton J.B., Bateman D.B., Telka A.M., Waller R., Whiteman C., Kuzmina S. 2017. Early to Mid Wisconsin Fluvial Deposits and Palaeoenvironment of the Kidluit Formation, Tuktoyaktuk Coastlands, Western Arctic Canada // Permafrost and Periglacial Processes. Vol.28. No.3. P.523-533.

Nelson R.E. 1982. Late Quaternary Environments of the Western Arctic Slope, Alaska. Ph.D. Thesis. Seattle: University of Washington. $149 \mathrm{p}$.

Nelson R.E. 1986. Mid-Wisconsin aridity in northern Alaska: incongruity of a mesic pollen record and xeric insect indicators // Curr. Res. Pleistocene. Vol.3. P.57-59.

Nelson R.E. 1998. Mid to Late Wisconsinan transition in Northern Alaska: contrasting pollen and macrofossil records from a fluvial section on the Titaluk River // Geol. Soc. Amer. Abstr. Progr. Vol.30. No.7. P.A-143.

Nelson R.E. 2001. Bioclimatic Implications and Distribution Patterns of the Modern Ground Beetle Fauna 
(Insecta: Coleoptera: Carabidae) of the Arctic Slope of Alaska, U.S.A. // Arctic. Vol.54. No.4. P.425-430.

Nelson R.E., Carter L.D. 1987. Paleoenvironmental analysis of insects and extralimital Populus from an early Holocene sites on the Arctic Slope of Alaska, USA // Arct. Alp. Res. Vol.19. P.230-241.

Nelson R.E., Carter L.D. 1997. Mid- to Late-Wisconsin transition in northern Alaska: contrasting loess and fluvial pollen records and implications for land bridge environments // J. Brigham-Grette, S. Elias (eds.). Beringian Paleoenvironments Workshop. Boulder: University of Colorado. P.76.

Orlova-Bienkowskaja M.Ya. 2001. Daphniidae: genus Simocephalus. Guides to the identification of the microinvertebrates of the continental waters of the World. Vol.17. Leyden: Backhuys. 130 p.

Robinson S., Beaudoin A.B., Froese D.G., Doubt J., Clague J.J. 2007. Plant Macrofossils Associated with an Early Holocene Beaver Dam in Interior Alaska // Arctic. Vol.60. No.4. P.430-438.

Schweger C.E. 1997. Late Quaternary Palaeoecology of the Yukon: A Review // H.V. Danks, J.A. Downes (eds.). Insects of the Yukon (Terrestrial Arthropods). Ottawa: Biological Survey of Canada. P.59-72.

Sher A.V. 1976. [The role of Beringian Land in the development of Holarctic Mammalian fauna in the Late Cenozoic] // V.L. Kontrimavichus (ed.). Paleoekologiya Beringii. Vladivostok: Far East Science Center AS USSR. P.227-241 [in Russian].

Sher A.V. 1974. Pleistocene mammals and stratigraphy of the far northeast USSR and North America // Intern. Geol. Rev. Vol.16. No.7-10. P.1-284.

Sher A., Kuzmina S. 2007. Beetle records/ Late Pleistocene of Northern Asia // S. Elias (ed.). Encyclopedia of Quaternary Science. Vol.1. Amsterdam: Elsevier. P.246-267.

Sher A.V., Kuzmina S.A., Kuznetsova T.V., Sulerzhitsky L.D. 2005. New insights into the Weichselian environment and climate of the Eastern-Siberian Artic, derived from fossil insects, plants, and mammals // Quaternary Sci. Rev. Vo.24. P.533-569.

Smith L.C., MacDonald G.M., Velichko A.A., Beilman D.W., Borisov O.K., Frey K.E., Kremenetsk, K.V., Sheng Y. 2004. Siberian peatlands a net carbon sink and global methane source since the early Holocene // Science. Vol.303. No.5656. P.353-356.

Ter-Minassian M.E. 1979. [Review of the weevil genus Stephanocleonus Motsch. (Coleoptera, Curculionidae)] // Nasekomye Mongolii. Leningrad. Vol.6. P.184-342 [in Russian]

Trimble D.A., Robinson S.W. 1989. U S Geological Survey, Menlo Park, California, Radiocarbon measurements, IV // Radiocarbon. Vol.31. No.1. P.69-84.

Yurtsev B.A. 1972. Phytogeography of north eastern Asia and the problem of Transberingian floristic interrelations // A. Graham (ed.). Floristics and Paleofloristics of Asia and Eastern North America. Amsterdam: Elsevier. P.19-54.

Yurtsev B.A. 2001. The Pleistocene "Tundra-Steppe" and the productivity paradox: the landscape approach // Quaternary Sci. Rev. Vol.20. P.165-174.

Zazula G.D., Froese D.G., Schweger C.E., Mathewes R., Telka A., Harington C.R., Westgate J.A. 2003. Late Pleistocene steppe macrofossils in east Beringia // Nature. Vol.423. P.603.

Responsible editor K.G. Mikhailov 